\title{
Polycrystalline graphene and other two-dimensional materials
}

\author{
Oleg V. Yazyev' and Yong P. Chen ${ }^{2}$
}

\begin{abstract}
Graphene, a single atomic layer of graphitic carbon, has attracted intense attention because of its extraordinary properties that make it a suitable material for a wide range of technological applications. Large-area graphene films, which are necessary for industrial applications, are typically polycrystalline - that is, composed of single-crystalline grains of varying orientation joined by grain boundaries. Here, we present a review of the large body of research reported in the past few years on polycrystalline graphene. We discuss its growth and formation, the microscopic structure of grain boundaries and their relations to other types of topological defect such as dislocations. The Review further covers electronic transport, optical and mechanical properties pertaining to the characterizations of grain boundaries, and applications of polycrystalline graphene. We also discuss research, still in its infancy, performed on other two-dimensional materials such as transition metal dichalcogenides, and offer perspectives for future directions of research.
\end{abstract}

T he field of research in two-dimensional (2D) materials has been enjoying extraordinary growth during the past decade. This activity was triggered by pioneering works on graphene $^{1-3}$, a $2 \mathrm{D}$ semimetallic allotrope of carbon that turned out to be an exceptionally fertile ground for advancing frontiers of condensed matter physics ${ }^{4-7}$. The centre of interest then rapidly shifted from fundamental science to potential technological applications of this $2 \mathrm{D}$ material $^{8-10}$. Furthermore, other atomically thin monolayer systems, which possess some valuable properties for many applications, soon joined the field, thus extending the palette of available 2D materials. Examples include insulating monolayer hexagonal boron nitride ( $\mathrm{h}-\mathrm{BN})^{11}$ and semiconducting transition metal dichalcogenides (TMDCs) $\mathrm{MX}_{2}(\mathrm{M}=\mathrm{Mo}, \mathrm{W} ; \mathrm{X}=\mathrm{S}, \mathrm{Se})$ characterized by electronic bandgaps between $1.1 \mathrm{eV}$ and $1.9 \mathrm{eV}$ (refs 12,13). The diversity of 2D materials further opens the possibility for such atomically thin crystals to be combined in complex heterostructures by stacking them on top of each other, thus giving rise to a whole new paradigm of nanoscale engineering ${ }^{7,14-16}$.

Technological applications require scalable techniques that would produce large-area sheets beyond the micrometre-size samples of graphene used in earlier research, such as single-crystalline graphene flakes readily obtained by mechanical exfoliation of graphite $^{1}$. Statistical physics arguments, however, suggest that crystalline order in 2D is highly susceptible to various types of fluctuation and disorder ${ }^{17}$, which would hinder production of high-quality singlecrystalline graphene sheets of arbitrarily large size. Practically, typical films of graphene of wafer scale or larger size as produced by, for example, chemical vapour deposition (CVD), are polycrystal$\operatorname{line}^{18-20}$ : that is, composed of single-crystalline domains of varying lattice orientation. In polycrystalline materials, such rotational disorder necessarily leads to the presence of grain boundaries interfaces between single-crystalline domains ${ }^{21,22}$. Grain boundaries (GBs) represent a class of topological defects - imperfections described by a structural topological invariant that does not change upon local modifications of the lattice ${ }^{23}$. Of course, such topological defects, intrinsic to polycrystalline materials, inevitably affect all properties of the material under study.

This Review discusses recent experimental advances in the emerging field of polycrystalline 2D materials, complemented with necessary theoretical concepts. We first cover recent progress in observing the micrometre-scale morphology in polycrystalline graphene as well as the atomic structure of GBs. The structure of the latter is explained in terms of hierarchical classification of topological defects in crystalline lattices. Special attention is devoted to peculiar behaviour of topological defects in graphene as opposed to those in bulk crystals. Next we cover important aspects of graphene growth by CVD for the formation of polycrystalline graphene. We then consider electronic transport, optical, mechanical and thermal properties, and related characterization techniques of polycrystalline graphene. The final section reviews several polycrystalline $2 \mathrm{D}$ materials other than graphene: monolayer h-BN, TMDCs and 2D silica. The Review is concluded with an outlook of future directions of research in this field.

\section{Structure of polycrystalline graphene}

The details of the structure of polycrystalline graphene at different length scales determine its properties. These various aspects of the structure down to atomic length scales have been investigated using several experimental techniques.

Experimental evidence. Historically, research on polycrystalline graphene was preceded by investigations of topological defects in bulk graphite. The first transmission electron microscopy (TEM) studies of dislocations in graphite were reported in the early $1960 \mathrm{~s}^{24,25}$. In 1966, Roscoe and Thomas proposed an atomistic model of tilt GBs in graphite, which suggested that the cores of edge dislocations are composed of pentagon-heptagon pairs ${ }^{26}$. This is consistent with the structure of topological defects in polycrystalline monolayer graphene discussed below. Later, the interest in GB defects in graphene was renewed with the advent of scanning tunnelling microscopy (STM) for investigating surfaces ${ }^{27-30}$. Scanning tunnelling spectroscopy (STS) allowed the local electronic properties of these defects in graphite to be investigated in detail ${ }^{31}$. The scanning probe techniques have also been used recently to explore the possible role of GBs in the intrinsic ferromagnetism of graphite ${ }^{32}$.

In bulk solids, the structure of dislocations and GBs is generally difficult to access and image using current microscopy techniques, as these defects are mostly buried deep inside. In contrast,

1Institute of Theoretical Physics, Ecole Polytechnique Fédérale de Lausanne (EPFL), CH-1015 Lausanne, Switzerland. ${ }^{2}$ Department of Physics and School of Electrical and Computer Engineering and Birck Nanotechnology Center, Purdue University, West Lafayette, Indiana 47907, USA.

e-mail: oleg.yazyev@epfl.ch; yongchen@purdue.edu 

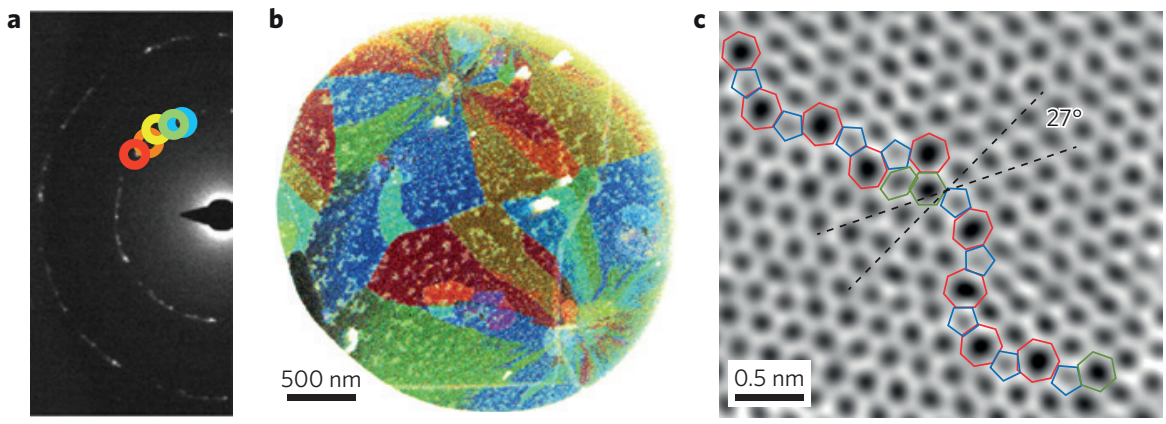

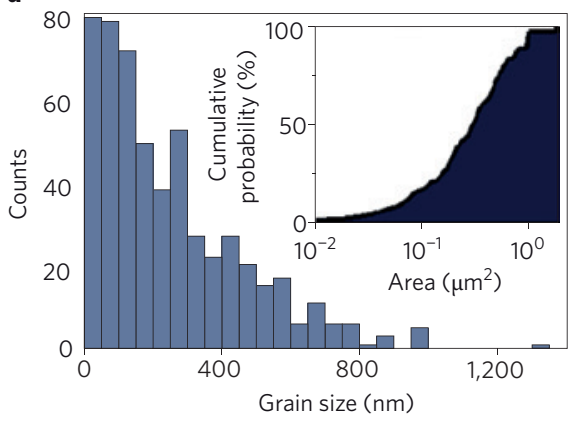

f

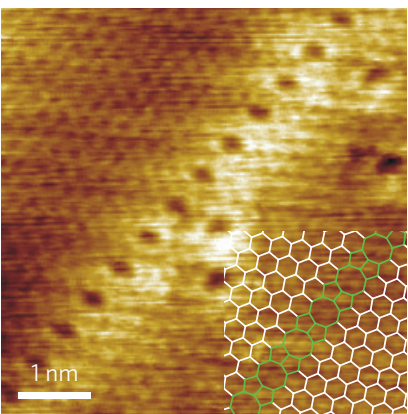

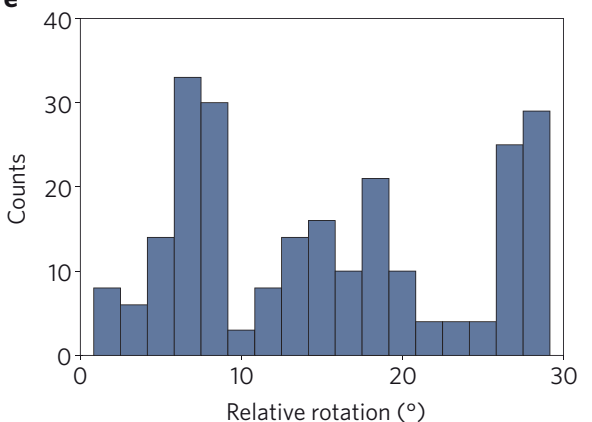

g

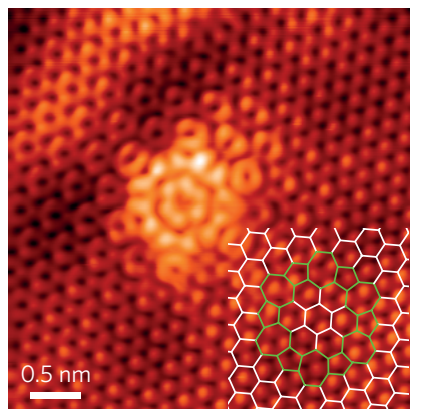

Figure 1 | Experimental studies of polycrystalline graphene and extended defects. a, Electron diffraction pattern from a sample of polycrystalline graphene showing numerous sets of six-fold-symmetric diffraction spots rotated with respect to each other. $\mathbf{b}$, False-coloured dark-field TEM image revealing individual single-crystalline graphene grains of varying shape, size and orientation. This image was constructed by aperturing the diffraction spots in a such that only the scattered electrons corresponding to one set of diffraction spots (colour-coded circles in a) are used to construct the realspace image. c, Aberration-corrected annular dark-field scanning TEM (ADF-STEM) image of a grain boundary stitching two graphene grains with lattice orientations rotated by $\sim 27^{\circ}$ with respect each other. The dashed lines outline the lattice orientations of the two domains. The structural model of the interface highlighting heptagons (red), hexagons (green) and pentagons (blue) is overlaid on the image. d,e, Distributions of grain sizes (d) and their relative orientations (e) in samples of polycrystalline graphene investigated in ref. 18. The inset shows the cumulative probability of having more than one grain given the area. f, STM image of a regular line defect in graphene grown on $\mathrm{Ni}(111)$ substrate ${ }^{45}$. The inset shows the structural model. $\mathbf{g}$, STM image of the flower-shaped point defect in epitaxial graphene grown on $\mathrm{SiC}(0001)^{48}$. Inset shows the structural model. Figure reprinted with permission from: a-e, ref. 18, Nature Publishing Group; f, ref. 45, Nature Publishing Group; g, ref. 48, (c) American Physical Society.

2D materials such as graphene provide an exceptional experimental system where such structural irregularities are exposed and can be studied in greater detail by microscopy, with resolution down to atomic levels, and even including temporal evolution. For polycrystalline graphene, TEM has become one of the most powerful and widely used tools to map out both the polycrystalline morphology on a large scale (above the size of single-crystalline grains), and the structural details of individual topological defects down to atomic scale ${ }^{33}$. Although early TEM observation of a dislocation in graphene was reported by Hashimoto et al. ${ }^{34}$ in 2004, the first systematic investigations of GBs in polycrystalline graphene were published only in 2011 $18-20$. These experiments were performed on graphene grown by CVD on copper substrate ${ }^{35}$. Huang et al. used diffraction-filtered dark-field (DF) TEM for large-area mapping of the location, size, orientation and shape of several hundred grains and grain boundaries ${ }^{18}$. In their study, individual crystalline orientations were isolated using an aperture to select the appropriate diffraction spot. The resulting images revealed an intricate patchwork of grains connected by tilt GBs (Fig. 1a-c). The grains in graphene samples produced by Huang et al..$^{18}$ are predominantly of submicrometre size (Fig. 1d), and GB misorientation angles show a complex multimodal distribution (Fig. 1e). The distribution of grain sizes and misorientation angles, however, depends strongly on the synthetic protocol used for producing graphene. For instance, An et al. reported a different distribution of misorientation angle, mostly confined between 10 and 30 degrees $^{20}$. 


\section{Box 1 | Relations between different types of topological defect in graphene.}

Polycrystalline materials are composed of single-crystalline domains with different lattice orientations. The changes of the lattice orientation are accommodated by the presence of topological defects. The structure of such defects is described by some topological invariant, a non-locally defined quantity conserved upon local structural transformations. There are three types of topological defect relevant to $2 \mathrm{D}$ materials - disclinations, dislocations and GBs - related to each other by hierarchical relations $s^{23,36,39}$. Importantly, in graphene these defects can be constructed without perturbing the native three-fold coordination sphere of $s p^{2}$ carbon atoms ${ }^{36}$.

Disclinations (a) are the elementary topological defects obtained by adding a semi-infinite wedge of material to, or removing it from, an ideal $2 \mathrm{D}$ crystalline lattice. For $60^{\circ}$ wedges, the resulting cores of positive $\left(s=60^{\circ}\right)$ and negative $\left(s=-60^{\circ}\right)$ disclinations are pentagons (red) and heptagons (blue), respectively, embedded into the honeycomb lattice of graphene. Wedge angle $s$ is the topological invariant of a disclination. The presence of isolated disclinations in graphene is unlikely as it inevitably results in highly non-planar structures.
Dislocations (b) are the topological defects equivalent to pairs of complementary disclinations. The topological invariant of a dislocation is the Burgers vector $\mathbf{b}$, which is a proper translation vector of the crystalline lattice. A dislocation effectively embeds a semiinfinite strip of material of width $\mathbf{b}$ into a $2 \mathrm{D}$ lattice ${ }^{36}$. An edgesharing heptagon-pentagon is a dislocation in graphene with the smallest possible Burgers vector equal to one lattice constant $(\mathbf{b}=(1,0))$. Larger distances between disclinations result in longer Burgers vectors, as illustrated by the $\mathbf{b}=(1,1)$ dislocation.

Grain boundaries (c) in 2D materials are equivalent to $1 \mathrm{D}$ chains of aligned dislocations ${ }^{38}$. These topological defects are the ultimate interfaces between single-crystalline grains in polycrystalline materials. The topological invariant of a GB in $2 \mathrm{D}$ is the misorientation angle $\theta=\theta_{\mathrm{L}}+\theta_{\mathrm{R}}\left(0^{\circ}<\theta<60^{\circ}\right)$, which is related to the density of dislocations and their Burgers vectors $\mathbf{b}$ via the so-called Frank's equations ${ }^{21}$. Large dislocation density (or, equivalently, small distance between the neighbouring dislocations) corresponds to large misorientation angles. Two examples of particularly stable large-angle $\mathrm{GBs}\left(\theta=21.8^{\circ}\right.$ and $\left.\theta=32.3^{\circ}\right)$ in graphene are shown.

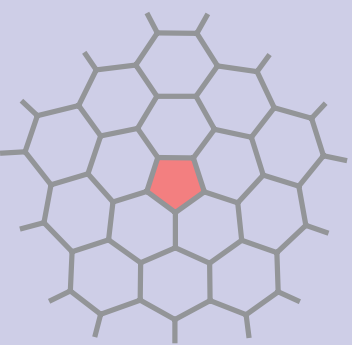

$s=60^{\circ}$

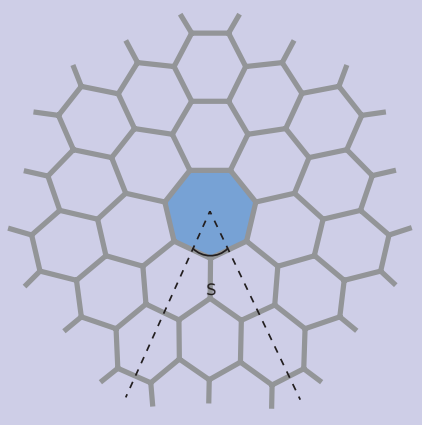

$s=-60^{\circ}$ b

Dislocations

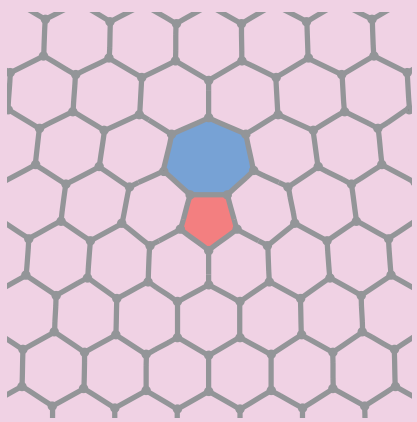

$\mathbf{b}=(1,0)$

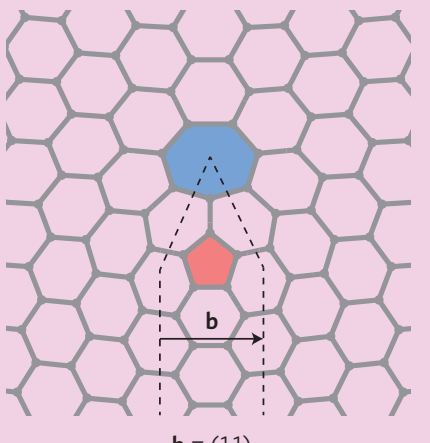

$\mathbf{b}=(1,1)$
Grain boundaries

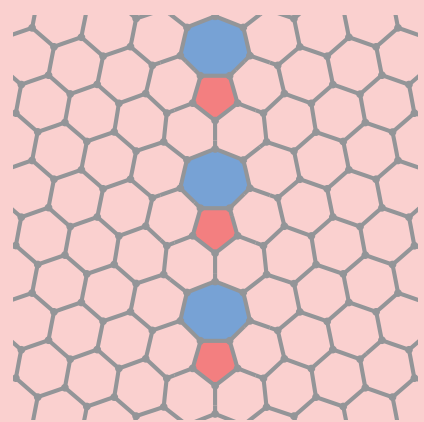

$\theta=21.8^{\circ}$

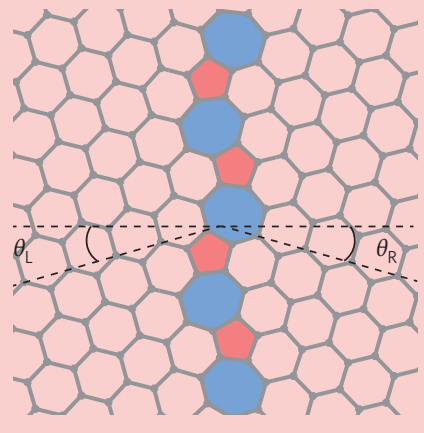

$\theta=32.3^{\circ}$
By using high-resolution TEM (HR-TEM), the details of atomicscale structure of GB defects were determined ${ }^{18,19}$. Many GBs exhibited atomically sharp interface regions formed by chains of alternating pentagons and heptagons embedded in the hexagonal lattice of graphene (Fig. 1c), in full agreement with previous theoretical predictions ${ }^{36,37}$. This structure can be understood in light of the Read-Shockley model ${ }^{38}$, which views tilt GBs as arrays of edge dislocations. Dislocations in graphene are represented by pairs of pentagons and heptagons (disclinations), the elementary structural topological defects in graphene. Hierarchical relations between the above-mentioned classes of structural topological defects ${ }^{23,36,37,39,40}$ and the definitions of their topological invariants are explained in Box 1. Importantly, this construction based on pentagonal and heptagonal units conserves the coordination environment of all carbon atoms, thus automatically resulting in energetically favourable structures. In contrast, the HR-TEM images by An et al. show the presence of undercoordinated atoms ('twinlike' structures) in the GB regions ${ }^{20}$, likely to be stabilized by adsorbates found in almost all of the boundary areas. Models of GBs containing undercoordinated carbon atoms, either with dangling bonds or forming complexes with extrinsic adsorbates, have been investigated theoretically ${ }^{41,42}$. Besides GBs involving interatomic bonds across the interface region, 


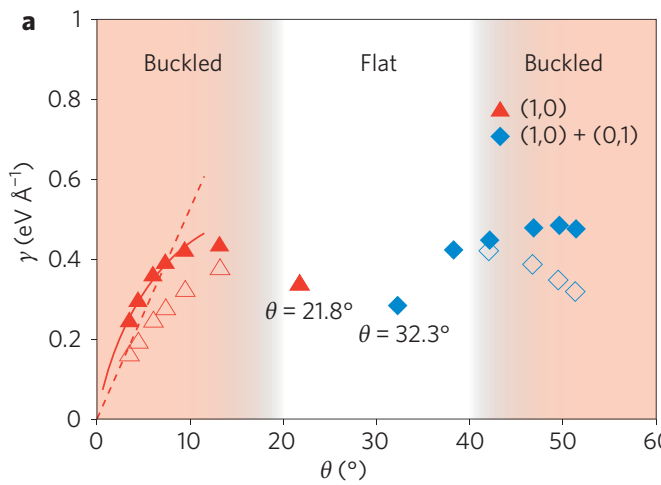

b

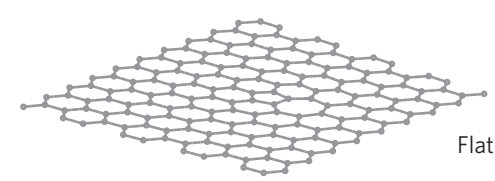

Flat

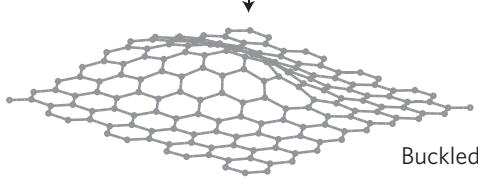

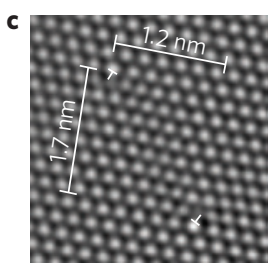

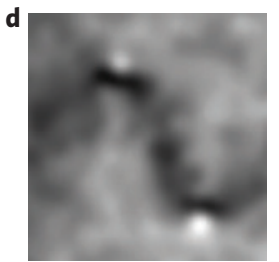

e
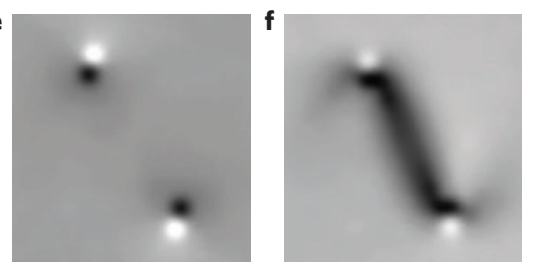

g

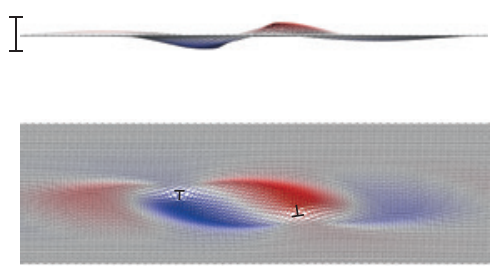

h Climb
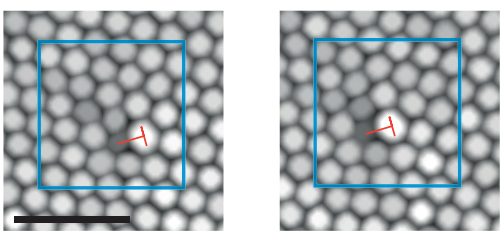

i Glide
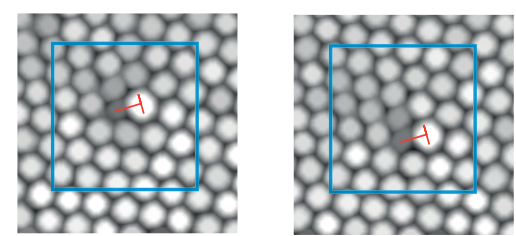

j Complex glide
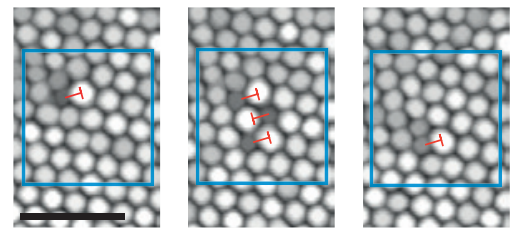

Figure 2 | Out-of-plane deformations and transformations of topological defects. a, Grain-boundary energies $\gamma$ plotted as a function of misorientation angle $\theta$ for symmetric defect configurations ${ }^{36}$. The colour of symbols reflects the Burgers vectors of constituent dislocations (red, $\mathbf{b}=(1,0)$ dislocations; blue, $\mathbf{b}=(1,0)+(0,1)$ dislocation pairs). The low-formation-energy, large-angle grain bounadries shown in the Box 1 figure, panel $\mathbf{c}$ are indicated by the corresponding values of the misorientation angle $\left(\theta=21.8^{\circ}\right.$ and $\left.\theta=32.3^{\circ}\right)$. Solid and open symbols correspond to flat and buckled configurations, respectively. Shaded areas indicate the ranges of misorientation angle in which the buckled configurations are energetically preferred over the flat ones. Solid and dashed lines correspond to the fits assuming the Read-Shockley equation and the finite formation energy ( $7.5 \mathrm{eV})$ of dislocations. $\mathbf{b}$, Transition to an out-of-plane corrugated state of graphene sheet produced by the presence of $\mathbf{a} \mathbf{b}=(1,0)$ dislocation. $\mathbf{c}, \mathrm{HR}-\mathrm{TEM}$ image of a pair of $\mathbf{b}=(1,0)$ dislocations in graphene separated by 1.2-nm glide distance and 1.7-nm climb distance. $\mathbf{d}$, Filtered image revealing the apparent in-plane compression (dark) and extension (bright). e,f, Simulated filtered images corresponding to flat (e) and buckled configurations (f). $\mathbf{g}$, Lowest-energy configuration of the corrugation produced by a pair of dislocations in relative arrangement similar to the one shown in c. Out-of-plane displacements of carbon atoms are colour-coded. $\mathbf{h}, \mathbf{i}$, Maximum filtered HR-TEM images reveal the dislocation climb (h) and glide (i) processes. $\mathbf{j}$, An observation of a complex glide process that starts with a bond rotation event next to the dislocation core and involves an intermediate aggregate of three dislocations. The positions of dislocation cores are indicated by red symbols. Blue boxes serve as a fixed reference. Scale bars in panels $\mathbf{g}, \mathbf{h}, \mathbf{j}: 1 \mathrm{~nm}$. Panels c-j reprinted from ref. 63, Nature Publishing Group.

several studies have also reported weakly connected GBs formed by 'overlapping' individual grains: that is, with one domain grown over the top of a neighbouring domain ${ }^{43,44}$.

Several examples of topologically trivial defects (that is, characterized by zero values of the relevant structural topological invariants) derived from GBs in graphene deserve special attention. Lahiri et al. reported an observation of highly regular line defects in graphene grown on $\mathrm{Ni}(111)$ substrate ${ }^{45}$. Such a one-dimensional defect formed by alternating octagons and pentagon pairs aligned along the zigzag direction (Fig. 1f) can be viewed as a degenerate GB as it has zero misorientation angle. Because of its topologically trivial structure, this defect can be engineered in a controlled way, as demonstrated by Chen et al. ${ }^{46}$. Another work observed a different line defect in graphene oriented along the armchair direction ${ }^{47}$. GB loops are formally equivalent to point defects in crystal lattices. A striking example is the highly symmetric flower-shaped defect found in graphene produced using different methods (Fig. 1g) ${ }^{48,49}$. Less-symmetric small GB loops have also been observed in TEM studies ${ }^{50-52}$.

Finally, a different type of topological defect is possible in multilayer systems such as bilayer graphene. Several groups have reported observations of boundaries between domains with structurally equivalent $\mathrm{AB}$ and $\mathrm{AC}$ stacking orders in bilayer graphene $\mathrm{e}^{53-57}$. These stacking domain boundaries observed by means of DF-TEM appear as regions of continuous registry shift that are a few-nanometres wide, and often form dense networks in bilayer graphene.
Grain boundary energies and out-of-plane deformations. Formation energies play a crucial role in determining the atomic structure of GBs at conditions close to thermodynamic equilibrium. This has been investigated theoretically using density functional theory $^{36}$ and empirical force fields ${ }^{37,58,59}$. Figure 2 a shows the computed GB energies $\gamma$ for a number of symmetric periodic configurations characterized by different values of misorientation angle $\theta$ (ref. 36). Two scenarios can be considered here. First, GBs are constrained to assume flat morphology when strong adhesion of graphene to a substrate takes place. In this case, the energetics of these defects (filled symbols in Fig. 2a) can be described by the Read-Shockley equation as for bulk materials (solid line in Fig. $2 \mathrm{a})^{21,38}$. The definition of misorientation angle $\theta$ given in Box 1 results in two small-angle regimes for which the distance $d$ between neighbouring dislocations forming the GB is larger than the length of their Burgers vectors $\mathbf{b}$. These regimes imply that $\gamma$ decreases as $d$ increases for $\theta \rightarrow 0^{\circ}$ and $\theta \rightarrow 60^{\circ}$, respectively. For intermediate values of $\theta$ the distance between neighbouring dislocations is comparable to their Burgers vectors (large-angle GBs). Importantly, this regime is characterized by a minimum in $\gamma(\theta)$ (Fig. 2a). The low formation energies of largeangle GBs are explained by efficient mutual cancellation of in-plane elastic strain fields induced by closely packed dislocations. In particular, the two regular GB configurations shown in Box 1 have especially low formation energies of 0.34 and $0.28 \mathrm{eV}^{-1}$, respectively, according to the results of first-principles calculations ${ }^{36}$. 
The situation of freely suspended graphene is remarkably different. Unlike bulk materials, the atoms of 2D graphene sheets are allowed to displace in the third, out-of-plane dimension. The possibility of out-of-plane displacement has profound effects on the energetics of topological defects in suspended graphene or graphene weakly bound to substrates. In particular, the out-of-plane corrugations effectively 'screen' the in-plane elastic fields produced by topological defects, thus greatly reducing their formation energies $^{23,39}$. Whereas large-angle GBs in suspended graphene are flat, the stable configurations of small-angle defects are strongly corrugated (open symbols in Fig. 2a). Moreover, the out-of-plane displacements lead to finite magnitudes of otherwise diverging formation energies of isolated dislocations. First-principles and empirical force-field calculations predict formation energies of $7.5 \mathrm{eV}$ (ref. 36) and $6.2 \mathrm{eV}$ (ref. 60), respectively, for an isolated $\mathbf{b}=(1,0)$ dislocation. Remarkably, these values are comparable to formation energies of simple point defects in graphene, for example the Stone-Wales defect $(4.8 \mathrm{eV})$ and single-atom vacancy $(7.6 \mathrm{eV})^{61}$. The corrugation profile produced by $\mathbf{a} \mathbf{b}=(1,0)$ dislocation appears as a prolate hillock (Fig. 2b), in agreement with the results of an STM study of dislocations in epitaxial graphene on $\operatorname{Ir}(111)$ substrate $^{62}$

Out-of-plane deformations induced by the presence of topological defects in graphene have been investigated using electron microscopy techniques ${ }^{63,64}$. In TEM, the corrugation fields are observed indirectly as apparent in-plane compressive strain due to the tilting effect of the graphene sheet. An example from ref. 63 considers the case of a pair of dislocations separated by about $2 \mathrm{~nm}$ (Fig. 2c). The filtered image reveals the presence of an extended region of compressive strain connecting the two dislocations (Fig. 2d). Atomistic simulations assuming a perfectly flat graphene layer show only the presence of two localized in-plane stress dipoles (Fig. 2e), whereas allowing for out-of-plane relaxation reproduces the experimentally observed region of apparent compression (Fig. 2f). A 3D view of the out-of-plane deformation profile produced by a pair of dislocations is shown in Fig. 2g.

Out-of-plane corrugation can also act as an efficient mechanism for relieving the misfit strain at asymmetric GBs in graphene. In this case, compressive strain was predicted to result in periodic ripples along the GB defects ${ }^{40,65}$. Such periodic ripples have recently been observed in a STM study of GBs on the surface of graphite $e^{66}$.

Transformations of topological defects. Understanding the transformation pathways of topological defects is important for describing its plastic deformation. The motion of individual dislocations in graphene has been observed using HR-TEM ${ }^{63,67}$. In accord with early theory predictions, the two basic steps of dislocation motion - glide and climb - are realized by means of a single $\mathrm{C}-\mathrm{C}$ bond rotation (the Stone-Wales transformation) (68,69 $^{6}$ and removal of two carbon atoms $s^{69,70}$, respectively. The energy barriers associated with these processes are high enough to render them unlikely under equilibrium conditions. For instance, the energy barrier of a bond rotation step was predicted to lie in the $5-10 \mathrm{eV}$ range ${ }^{61}$. Even higher energy barriers are expected for the sputtering of carbon atoms ${ }^{71,72}$. Under TEM conditions, however, irradiation by high-energy electrons at accelerating voltages close to the displacement threshold $(80 \mathrm{kV}$ in refs 63,67 ) promotes the above-mentioned elementary processes of dislocation motion. In particular, both dislocation climb (Fig. 2h) and glide (Fig. 2i) have been observed. A complex glide process with an intermediate configuration involving an aggregate of three dislocations has also been evidenced (Fig. $2 \mathrm{j}$ ).

Transformation of large-angle $\left(\theta \approx 30^{\circ}\right)$ GBs described as nearly continuous chains of pentagon-heptagon pairs has also been investigated using TEM $^{52}$. According to a simple thermodynamic argument, one expects a GB line to evolve only in the presence of significant boundary curvature. Indeed, nearly straight GBs showed fluctuating transformations without any time-averaged translation of the boundary line. In contrast, closed GB loops were shown to shrink under the electron irradiation, leading to complete elimination of small graphene grains fully enclosed within another single-crystalline domain.

\section{CVD growth of polycrystalline graphene}

Although there are numerous ways to produce graphene, CVD on polycrystalline $\mathrm{Cu}$ foils ${ }^{35}$ has now become the most widely used method to synthesize high-quality, large-size monolayer graphene films because of its simplicity, low cost and scalability. This technique produces the largest (over a metre so $\mathrm{far}^{73}$ ) graphene sheets that can be easily transferred to other substrates for diverse applications. The vast majority of experimental studies on GBs in graphene have been performed on such CVD-grown samples. In such a CVD growth, thermal decomposition of hydrocarbon gas (most commonly $\mathrm{CH}_{4}$, mixed with $\mathrm{Ar}$ and $\mathrm{H}_{2}$ ) at high temperature provides the source of carbon atoms that will ultimately assemble into graphene on the surface of $\mathrm{Cu}$ substrate. Details of this process are subject to much research and are believed to involve multiple steps and intermediates ${ }^{74,75}$. Single-crystalline graphene grains nucleate around multiple spots (the nucleation centres) on the substrate, grow in size, and as the growth proceeds, eventually merge to form a continuous polycrystalline graphene. Its properties will be determined by the constituent grains (their size, shape, edge orientation and other properties) and how they are merged or stitched together (that is, the structure of GBs).

By stopping the growth before all the grains merge into a continuous polycrystalline film, single crystal grains (as well as isolated GBs between two grains) can be obtained ${ }^{35,76,77}$, allowing the formation and properties of these building blocks of polycrystalline graphene to be studied. The polycrystallinity of the $\mathrm{Cu}$ foil is not a limiting factor for single-crystalline graphene growth, as a graphene grain can grow across GBs in Cu (Fig. 3a,b). This indicates weak interaction between graphene and $\mathrm{Cu}$ surfaces with no clear epitaxial relationship ${ }^{76,77}$. On the other hand, such interactions still exist, and $\mathrm{Cu}$ crystal orientation can still have some influence on the growth of the graphene overlayer ${ }^{78-83}$. Imperfections (defects, GBs and surface steps) and impurities in the $\mathrm{Cu}$ substrate can provide the nucleation centres for growth ${ }^{79,84}$. Recently, it was discovered that the presence of oxygen on the $\mathrm{Cu}$ surface can substantially decrease the graphene nucleation density by passivating $\mathrm{Cu}$ surface active sites (Fig. 3c $)^{77}$. Reducing the density of nucleation centres is the key to growing large single crystals of graphene ${ }^{77,85-87}$. Nucleation can also be artificially started using growth seeds $s^{76,88}$.

Changing various growth parameters can control both the size and shape of graphene grains. For instance, grain size can be tuned by varying the growth rate $e^{44}$. Earlier studies noted that different CVD growth pressures can lead to different grain shapes, with the two most common being flower-shaped grains often obtained in low-pressure $\mathrm{CVD}^{35,76}$ and hexagonal grains in atmospheric-pressure CVD (Fig. 3a,b) ${ }^{76}$. The flower-like (dendritic) shape ${ }^{35}$, with irregular and multifractal-like edges ${ }^{35,89,90}$, indicates a diffusionlimited growth mechanism. The more regular hexagonal grains ${ }^{76}$, whose edges are shown to be predominantly oriented along the zigzag directions of graphene lattice ${ }^{76,91}$, represent an edge-attachmentlimited growth $\mathrm{h}^{77}$. Hydrogen plays an important role by serving as an activator of the surface-bound carbon needed in graphene growth as well as an etching reagent that controls the size and morphology of the graphene grains ${ }^{92}$. The shape and size of the grains can be continuously tuned by hydrogen partial pressure (Fig. 3d,e $)^{90,92}$. Oxygen also accelerates graphene grain growth and shifts the growth kinetics from edge-attachment-limited (hexagonal-shaped grains) to diffusion-limited (dendritic-shaped grains) by reducing 
a

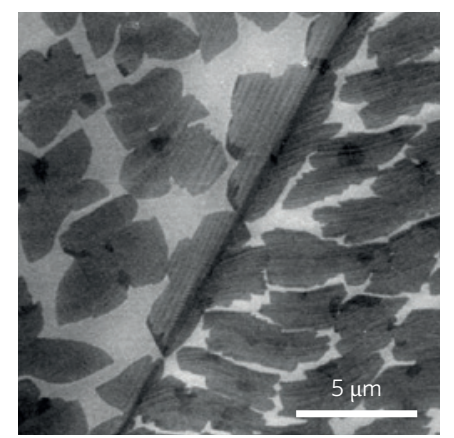

b

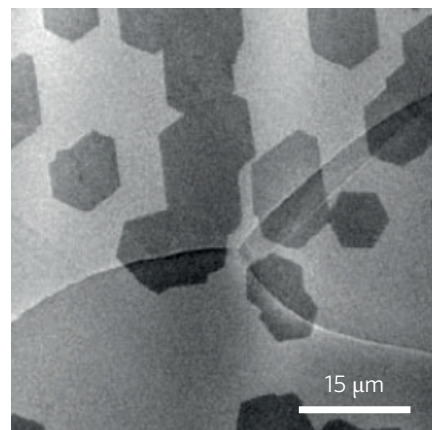

d
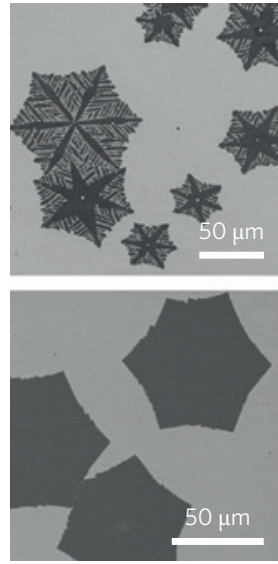
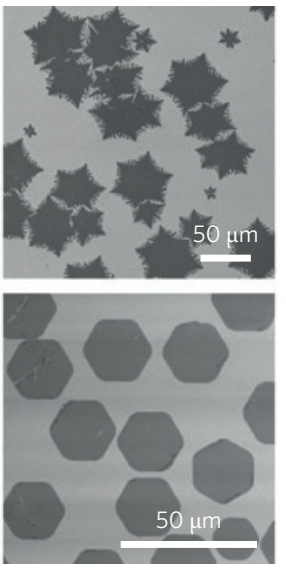
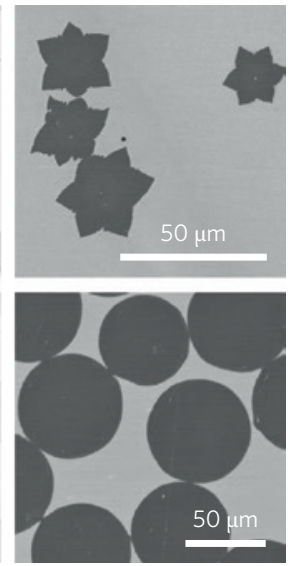

c
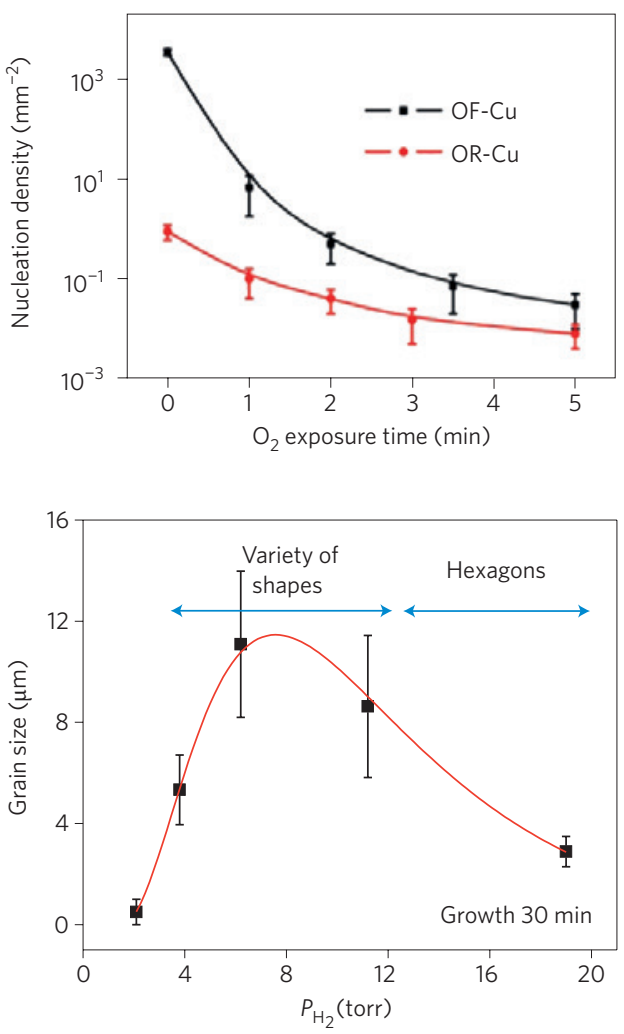

Figure 3 | Growth of graphene grains and polycrystalline graphene by chemical vapour deposition. $\mathbf{a}, \mathbf{b}$, SEM images of flower-shaped and hexagonalshaped graphene grains nucleated from different locations on Cu foil in low-pressure CVD (a) and atmospheric-pressure CVD (b) growth, stopped before the grains merge into a continuous sheet of polycrystalline graphene. It is seen that graphene grains can grow continuously across Cu grain boundaries on polycrystalline $\mathrm{Cu}$ foils. c, Nucleation density of graphene grains measured as a function of $\mathrm{O}_{2}$ exposure time on oxygen-free (OF-Cu) and oxygen-rich (OR-Cu) Cu foils demonstrating the effect of $\mathrm{O}_{2}$ in reducing nucleation density and increasing the grain size ${ }^{77}$. $\mathrm{P}_{\mathrm{CH} 4}=1 \times 10^{-3}$ Torr. $\mathbf{d}, \mathrm{SEM}_{\mathrm{images}}$ showing the evolution of the shape of graphene grains (from snowflake or flower-like, to hexagonal, and even circular) with varying growth conditions (mainly

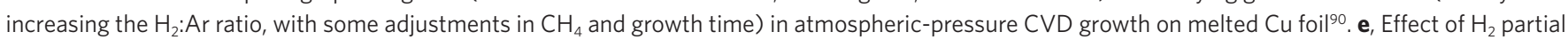
pressure during CVD growth on the size and shape of graphene grains, where flower-like or irregular shapes are obtained at low $\mathrm{H}_{2}$ partial pressures, and hexagonal shapes at high pressures ${ }^{92}$. Similar phenomena are observed in both atmospheric-pressure CVD and low-pressure CVD. Figure reprinted with

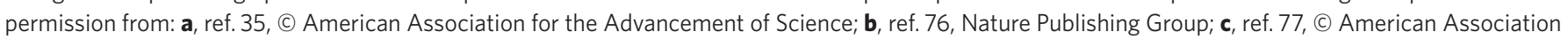
for the Advancement of Science; d, ref. 90, Nature Publishing Group; e, ref. 92, (C) American Chemical Society.

the activation barrier of the rate-limiting step ${ }^{77}$. The shape of grain is also affected by growth temperature ${ }^{93}$. In a growth mechanism model developed in ref. 77 , the shape is controlled by the balance between the characteristic time of carbon attachment and carbon flux, with the longer attachment time favouring hexagonal shapes. Understanding the reactivity and kinetics of graphene edges is critical for understanding the growth mechanism ${ }^{94,95}$.

\section{Optical imaging and characterization}

It is generally difficult for conventional optical imaging to visualize GBs in graphene directly, as they typically have very narrow widths (far smaller than optical resolution) and do not have sufficient optical contrast with the surrounding graphene grains. Most GBs are also quite flat and can be difficult to see even with scanning electron microscopy (SEM) and atomic force microscopy (AFM) ${ }^{76}$. However, it is possible to use auxiliary agents that have characteristic interactions with graphene grains or GBs to aid in optical imaging. For example, Kim et al. developed a simple method to visualize graphene grains and GBs by imaging the birefringence of a graphene surface covered with nematic liquid crystals, taking advantage of the orientation of the liquid crystals with the underlying graphene lattice (thus mapping the graphene grains and GBs to those of the corresponding liquid crystal domains $)^{96}$. Using optical microscopy, one can also enhance the contrast of grains and make GBs more visible by taking advantage of the more effective oxidation of substrate $(\mathrm{Cu})$ in the region exposed or under GBs (which can be further functionalized to facilitate oxidation $)^{97,98}$. Similar techniques can also be used to facilitate AFM and other types of GB imaging ${ }^{99}$.

Beyond standard optical imaging, GBs can be easily visualized using spectroscopic Raman imaging of the defect-activated D mode $\left(\sim 1,350 \mathrm{~cm}^{-1}\right)^{76,97}$. This also demonstrates strong inter-valley scattering of charge carriers at GBs (as such scattering is a part of the $\mathrm{D}$ peak Raman process) and corroborates the electronic transport measurements (for example weak localization) of $\mathrm{GBs}^{76}$. It is also possible to visualize and investigate electronic properties of GBs using an infrared nano-imaging technique, as demonstrated in ref. 100. The technique utilizes surface plasmons that are reflected and scattered by GBs, thus causing plasmon interference patterns that are recorded to map out the GBs. Further quantitative analysis of such images reveals that GBs form electronic barriers (with effective width $\sim 10-20 \mathrm{~nm}$, of the order of the Fermi wavelength in graphene and dependent on electronic screening) that obstruct both electrical transport and plasmon propagation ${ }^{100}$. Raman and plasmonic-based imaging do not require any auxiliary agents to treat the graphene. 


\section{Transport phenomena}

The electronic transport properties of polycrystalline graphene are certainly among the most important, considering the potential applications of large-area graphene in electronics. Many experiments have shown, consistent with intuition, that GBs can impede electronic transport, thus degrading the conductive properties of polycrystalline graphene compared with single-crystalline graphene $e^{76,101-104}$. For example, mobility measurements of polycrystalline graphene samples showed that larger grain sizes generally lead to higher electronic mobilities, despite substantial fluctuations in the values from sample to sample ${ }^{101}$, while mobilities (either from Hall ${ }^{76,105}$ or field-effecttransistor $^{102}$ measurements) of single-crystalline CVD graphene without GBs can exceed 10,000 $\mathrm{cm}^{2} \mathrm{~V}^{-1} \mathrm{~s}^{-1}$ and be comparable with those of exfoliated graphene $\mathrm{e}^{106}$. Transport measurements on isolated individual GBs have shown that GBs generally result in higher electrical resistance (Fig. 4a,b), although the increase of resistance can vary across different $\mathrm{GBs}^{76,105}$. On the other hand, a few other experiments ${ }^{18,44,107}$ observed no significant effects on the conductive properties caused by GBs or variation of grain sizes, and some GBs that may even enhance the conduction (for example GBs involving 'overlapping' graphene layers ${ }^{44}$, Fig. $4 \mathrm{~d}$ ). These different experimental results can be reconciled by considering that polycrystalline graphene samples may contain many types of GBs with different effects on electronic transport. Furthermore, when interpreting experimental results, one should keep in mind that typical graphene samples may contain many other types of disorder (such as point defects, contaminants and nearby impurities) that can scatter electrons and lower the mobility, thus masking the effect of GBs.

Further transport measurements addressing dependence on temperature $\mathrm{e}^{102}$ and/or magnetic field ${ }^{76,105}$ have generated important insights into conduction and carrier-scattering mechanisms in polycrystalline graphene and helped in understanding how GBs affect transport. For example, magnetotransport measurements across isolated GBs in comparison with those performed on single-crystalline grains have shown that GBs give rise to prominent weak-localization (Fig. 4c) effects ${ }^{76,105}$. This indicates that GBs induce strong intervalley scattering of carriers, consistent with the expected lattice disorder associated with GBs and with the observed strong Raman D peak (whose activation also requires intervalley scattering ${ }^{108,109}$ ) for $\mathrm{GBs}^{76}$. Interestingly, the 'half-integer' quantum Hall effect (QHE), which is a hallmark of Dirac fermion transport in graphene, is maintained for the transport across $\mathrm{GBs}^{76,105}$. A welldeveloped QHE has been observed in polycrystalline samples as large as $1 \mathrm{~cm}$ in size ${ }^{110}$.

Various scanning probe techniques have been employed as powerful tools to investigate the local electronic properties of GBs, down to the atomic scale, providing detailed insight into the microscopic mechanism of how GBs affect electronic transport. For example, both STM (including associated STS that probes the differential conductance $\mathrm{d} I / \mathrm{d} V$ and the local electronic density of states) ${ }^{103}$ and conductive $\mathrm{AFM}^{104}$ measurements have demonstrated suppressed conductivity of GBs. Multiple-probe STM measurements have found that the resistance of a GB changes with the width of the disordered transition region between adjacent grains ${ }^{111}$. Furthermore, STM revealed that GBs give rise to standing wave patterns $^{112}$ (Fig. 4e) propagating along the zigzag direction with a decay length of $\sim 1 \mathrm{~nm}$. This observation is indicative of backscattering and inter-valley scattering processes (which were also observed at armchair edges in graphene ${ }^{91}$ ), thus corroborating the Raman and weak-localization measurements ${ }^{76,105}$. In addition, STM/STS measurements have found that GBs tend to be more $n$-type doped ${ }^{103,112}$ compared with the surrounding graphene, which is often found to be p-type doped owing to surface adsorbates and contaminants. This leads to the formation of $\mathrm{p}-\mathrm{n}$ junctions with sharp interfaces on the nanometre scale ${ }^{103}$.
On the theory side, remarkable predictions have been reported for the periodic models of GBs investigated using the LandauerBüttiker formalism ${ }^{113}$. Yazyev and Louie have shown that all periodic GBs can be divided into two distinct classes ${ }^{65}$. GB configurations belonging to the first class typically show very high probabilities of the transmission of low-energy charge carriers across the GB. Members of the second class, however, are characterized by significant transport gaps resulting in complete reflection of charge carriers in rather broad energy ranges (up to $\sim 1 \mathrm{eV}$ ) around the charge-neutrality point (see Fig. $4 \mathrm{f}$ for schematic illustration of the effect). This striking transport behaviour can be exploited in nanometre-scale electronic devices based on engineered GBs in graphene. Gunlycke and White have shown for the example of the structure described in ref. 45 that transmission of charge carriers across periodic line defects at oblique angles can lead to their strong valley polarization (Fig. $4 \mathrm{~g})^{114}$. This prediction suggests that engineered line defects can be used as components of future valleytronic devices based on graphene ${ }^{115-117}$.

The Landauer-Büttiker formalism has also been used for investigating the effect of strain ${ }^{118}$ and chemical functionalization ${ }^{119,120}$ on the transport properties of GBs in graphene. Electronic transport across disordered grain boundaries has been studied with the help of wavepacket evolution techniques, either locally ${ }^{121}$ or by employing realistic large-scale models of polycrystalline graphene ${ }^{122}$. The latter work revealed a simple linear scaling of the mean free path and conductivity with the average size of single-crystalline domains. Several groups have proposed transport models based on the potential variation in the GB region. In particular, the effect of GBs on transport mobility was qualitatively explained using a potential barrier model ${ }^{102}$. A quantitative model of boundary resistance developed in ref. 111 suggested an increased electron Fermi wave vector within the boundary region, possibly owing to boundary-induced charge-density variation. Finally, the topological nature of intrinsic defects of polycrystalline graphene bears important implications for its electronic transport properties ${ }^{123}$. This effect of dislocations can be accounted for by means of a gauge field ${ }^{124,125}$ that gives rise to localized states at the Dirac point ${ }^{126}$. These localized states, in turn, result in resonant backscattering of low-energy charge carriers, with the effect being especially strong for well-separated dislocations (that is, those belonging to small-angle GBs) ${ }^{123}$.

\section{Mechanical properties}

In bulk materials, the presence of defects usually leads to significant reductions of the tensile strength ${ }^{127}$. Knowing how dislocations and GBs affect mechanical properties of graphene is particularly important considering that (i) single-crystalline graphene is the strongest known material ${ }^{128}$ and (ii) in low-dimensional materials the effect of disorder is expected to be amplified. In their recent systematic study, Lee et al. addressed mechanical properties of CVD-grown polycrystalline graphene ${ }^{129}$. Their technique relies on nanoindentation measurements of suspended graphene samples in an AFM set-up, as illustrated in Fig. 5a. Elastic stiffness of polycrystalline graphene samples characterized for different grain sizes $(1-5 \mu \mathrm{m}$ and $50-200 \mu \mathrm{m}$ ) was found to be statistically identical to that of single-crystalline graphene. Figure $5 \mathrm{~b}$ reproduces the histogram of the ensemble of AFM nanoindentation measurements performed on large-grain $(50-200 \mu \mathrm{m})$ samples. The obtained elastic modulus of $339 \pm 17 \mathrm{~N} \mathrm{~m}^{-1}$ corresponds to a 3D Young's modulus of $\sim 1 \mathrm{TPa}$. The measured fracture loads of large-grain polycrystalline samples were also found to be statistically identical to those of pristine single-crystalline graphene (Fig. 5c). However, sizeable reduction of the mean value and broader distribution of fracture loads were observed for small-grain $(1-5 \mu \mathrm{m})$ samples. This implies that the strength of polycrystalline graphene is affected by randomly occurring structural defects. Indeed, nanoindentation measurements 
a

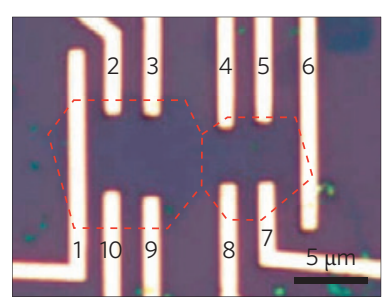

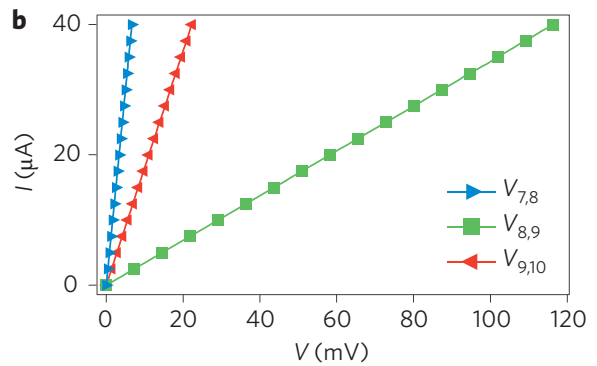

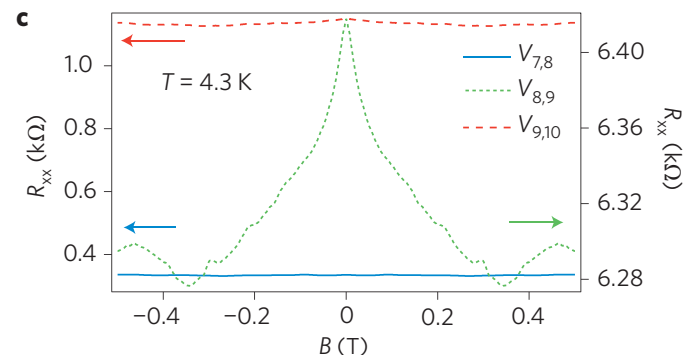

d

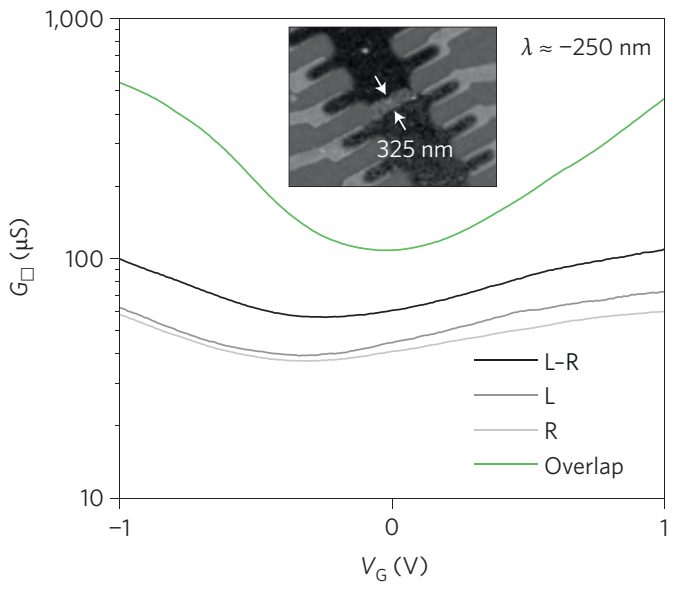

e
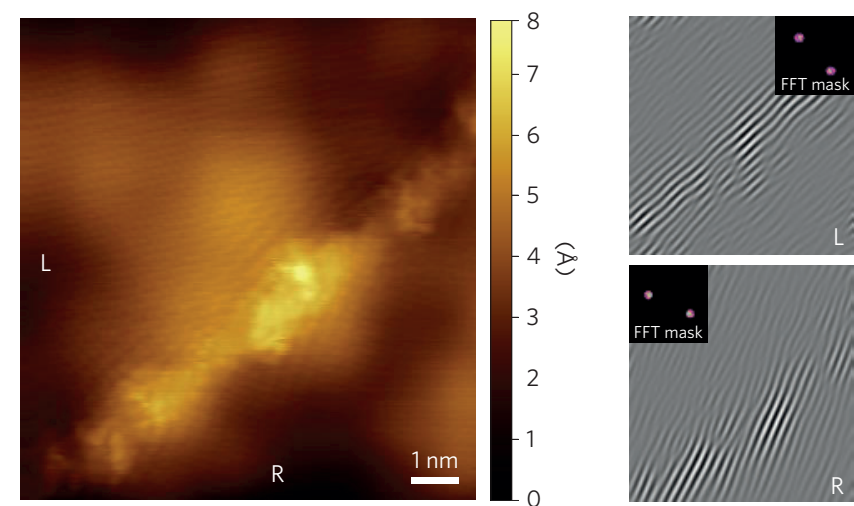

f

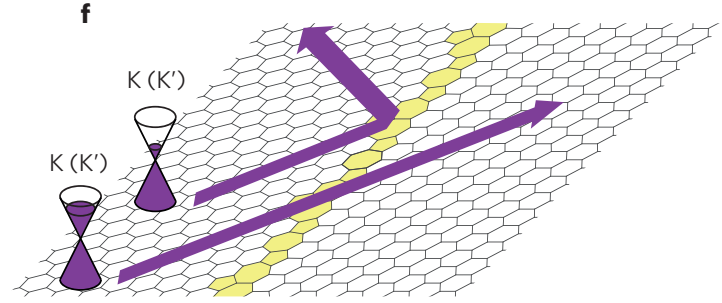

g

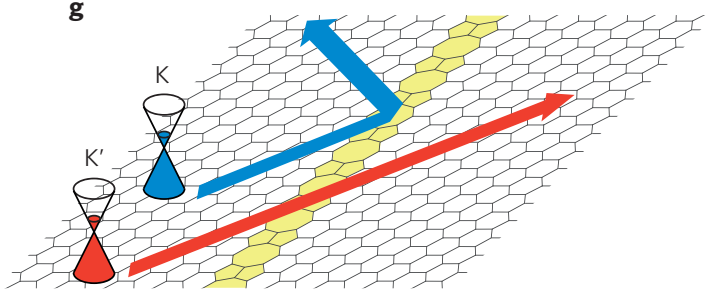

Figure 4 | Electronic transport across grain boundaries in graphene. a, Image of the device from ref. 76 with the red dashed line indicating the contour of two coalesced graphene grains with a GB in between. b, Room-temperature current-voltage (I-V) curves measured across an isolated GB (green) and within the graphene grain on each side of the GB (red and blue). The indices refer to contact numbers in $\mathbf{a}$. This measurement demonstrates a significantly reduced conductance (slope of the $I-V$ curve) of cross-GB transport compared with intragrain transport ${ }^{76}$. c, Low-temperature magnetoresistance measured across the GB and within the grains for the device shown in $\mathbf{a}$. The measurement reveals weak localization behaviour indicative of intervalley scattering caused by the GB. d, Gate-voltage $\left(V_{G}\right)$-dependent sheet conductance $\left(G_{\square}\right)$ measured across an 'overlapped' GB (labelled L-R) and within the grain on each side of the $G B(L$ and $R$ ), showing that such a GB actually enhances the conductance (or effectively 'shortens' the transport path length, in this case by $\lambda \approx-250 \mathrm{~nm})^{44}$. The conductance contributed by the GB is extracted (green). Inset: image of the device with the width of the 'overlap' region indicated $(325 \mathrm{~nm}$ ). e, STM image around a GB, revealing a linear structure (more clearly seen in the insets to the right showing the Fourier-filtered images corresponding to the left $(\mathrm{L})$ and right $(\mathrm{R})$ domains) that is associated with $\mathrm{GB}$-induced intervalley scattering ${ }^{112}$. $\mathbf{f}$, Schematic illustration of the transport gaps predicted for certain periodic GB defects discussed in ref. 65. Whereas low-energy charge carriers (upper Dirac cone drawing) are perfectly reflected, transmission across the GB defect is enabled at higher energies (lower Dirac cone drawing). $\mathbf{g}$, Schematic illustration of the valley filtering effect predicted for the line defect reported in ref. 45. At oblique incidence angles, charge carriers transmitted across the line defect acquire strong valley polarization. Charge carriers in valley K (blue) are preferentially reflected while those in valley $\mathrm{K}^{\prime}$ (red) are mostly transmitted across the line defect. The structures of defects in $\mathbf{f}, \mathbf{g}$ are emphasized with yellow filling of non-hexagonal rings. Figure reprinted with permission from: $\mathbf{a}, \mathbf{b}, \mathbf{d}$, ref. 76, Nature Publishing Group; c, ref. 44, (c) American Association for the Advancement of Science; e, ref. 112, (c) American Chemical Society.

performed directly on GB showed $20-40 \%$ smaller fracture loads than those performed in the middle of single-crystalline domains (Fig. 5d). Investigation of graphene membranes after indentation shows that cracks propagate not only along GBs but also inside grains (Fig. 5e). These results suggest that the elastic stiffness and strength of polycrystalline graphene with well-stitched GBs are close to those of pristine graphene. Importantly, the study also brings to one's attention the fact that post-growth processing techniques used in the previous studies ${ }^{130}$ significantly degraded the strength of graphene.
On the theory side, a number of computational studies of mechanical properties of polycrystalline graphene have been reported. Simulations performed on single GB models reveal that large-angle defect configurations (like those shown in the right column of Box 1) are as strong as pristine graphene, while smallangle defects characterized by larger interdislocation distances are significantly weaker ${ }^{131,132}$. We note that a recent nanoindentation study by Rasool et al. shows a clear reduction of the fracture force when small-angle GBs are probed ${ }^{133}$. The computational studies also provide an insight into the atomistic picture of mechanical failure 
a

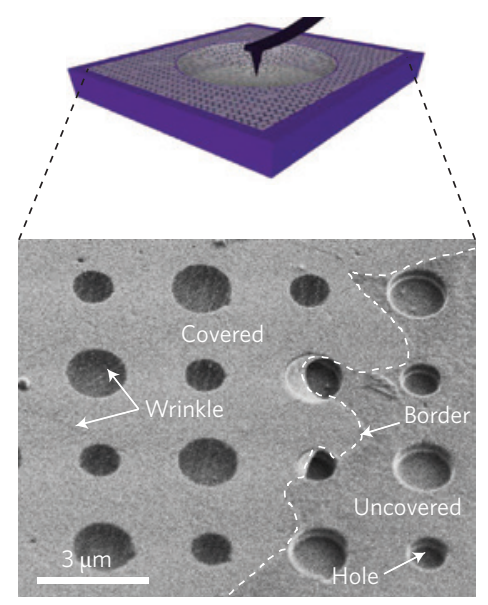

b

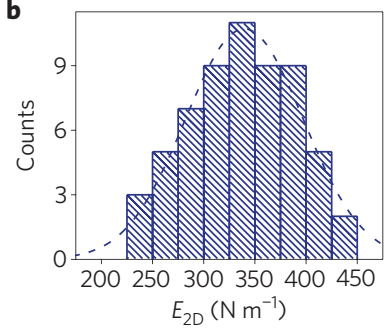

d

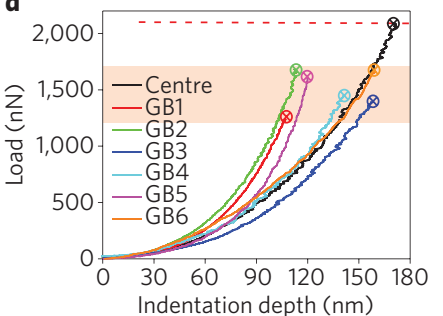

c

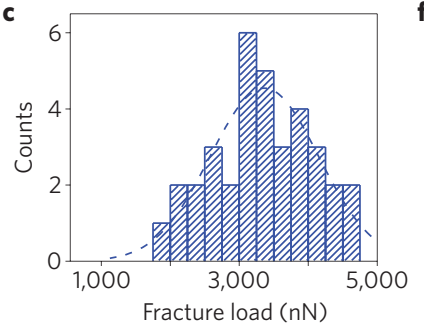

e

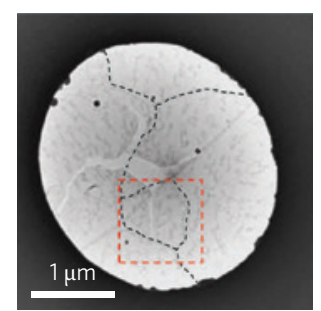

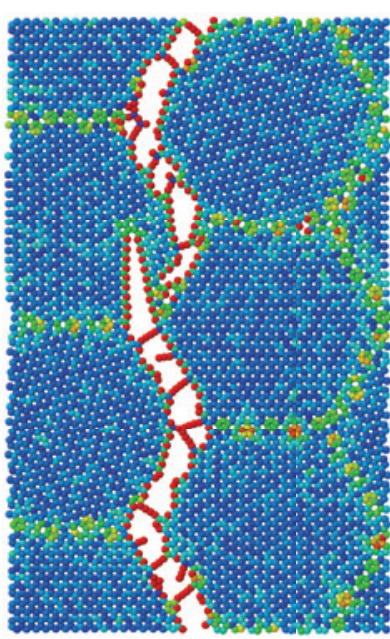

Figure $\mathbf{5}$ | Mechanical properties of polycrystalline graphene. a, Scheme of an AFM nanoindentation set-up for probing mechanical properties of suspended graphene ${ }^{129} \cdot \mathbf{b}, \mathbf{c}$, Histograms of elastic stiffness $\left(E_{2 \mathrm{D}}\right)(\mathbf{b})$ and fracture load $(\mathbf{c})$ measured for polycrystalline graphene samples characterized by a large grain size $(50-200 \mu \mathrm{m})$. The dashed lines represent fitted Gaussian distributions. d, Force-displacement curves for measurements performed on the GB and in the middle of a single-crystalline domain. The shaded area shows the range of fracture loads measured on the GBs, and the dashed line shows the fracture load measured in the middle of a single-crystalline domain. $\mathbf{e}$, Bright-field TEM image of cracks in suspended graphene after the nanoindentation experiment. The black dashed lines indicate the location of GBs before the nanoindentation experiment. The red square outlines the area in which intragranular crack propagation occurs. $\mathbf{f}$, Snapshot of a molecular dynamics simulation of the fracture process performed on an atomistic model of polycrystalline graphene ${ }^{135}$. The distribution of local tensile and compressive stress is shown in red and blue, respectively. The simulations reveal fracture via propagation of both inter- and intragranular cracks. Figure reprinted with permission from: a-e, ref. 129, (c) American Association for the Advancement of Science; $\mathbf{f}$, ref. 135, (c) American Chemical Society.

of polycrystalline graphene. In particular, several recent works reported simulations performed on models containing realistic $\mathrm{GB}$ networks ${ }^{134-136}$. It was found that triple junctions of GBs serve as nucleation centres for cracks. Propagation of cracks both along GBs and within single-crystalline grains was observed (Fig. 5f) ${ }^{134,135}$. Importantly, the failure of graphene can be considered as brittle, since dislocations are completely immobile at normal conditions $s^{135,136}$.

In addition to mechanical properties, it is also interesting to consider whether and how GBs may affect or even be used to engineer phonon (thermal) transport ${ }^{137,138}$. This remains a largely unexplored topic, calling for much further study, especially experiments. The possible different ways in which GBs may affect thermal transport compared with electronic transport could promise interesting functionalities, for example for thermoelectric devices.

\section{Topological defects in other 2D materials}

From a structural point of view, monolayer h-BN and TMDCs are closely related to graphene (Fig. 6a). Monolayer h-BN has the same honeycomb crystal structure as graphene, with a similar lattice constant $(a=2.50 \AA$ ) and two sublattices populated by B and $\mathrm{N}$ atoms, respectively. Such polarity of the crystalline lattice leads to profound consequences when atomic structure of topological defects is considered. In particular, odd-membered rings present in the topological defects break the alternating order of the two atomic species, resulting in homoelemental bonding ${ }^{139}$. The presence of covalent bonds between atoms of the same charge increases formation energies of defects and introduces an extra degree of freedom in defining their structures. For example, the $\mathbf{b}=(1,0)$ dislocations now come in two different flavours featuring either a $\mathrm{B}-\mathrm{B}$ bond or an $\mathrm{N}-\mathrm{N}$ bond (Fig. 6b). The two possible structures of dislocation cores are characterized by local deviation from the nominal stoichiometry (B-rich and N-rich) and by local charges (positively and negatively charged dislocations, respectively). Alternatively, dislocation cores involving only even-membered rings would allow the formation energy penalty associated with homoelemental bonding to be avoided. An example of such a neutral dislocation core composed of an edgesharing pair of four- and eight-membered rings is shown in Fig. 6 b. However, this dislocation is characterized by a larger $\mathbf{b}$ of $(1,1)$, implying a significantly higher contribution to the formation energy due to the elastic field. Available experimental evidence speaks in favour of defect structures containing homoelemental bonds. An atomic-resolution TEM image of a GB in monolayer h-BN shows three dislocations with cores composed of pentagon-heptagon pairs, two of which are B-rich and one N-rich (Fig. 6c) ${ }^{140}$.

The crystal structure of monolayer TMDCs is composed of a triangular lattice of metal atoms sandwiched between two triangularly packed planes of chalcogen atoms. In the most stable $2 \mathrm{H}$ phase, chalcogen atoms are stacked along the direction orthogonal to the monolayer. The crystal structure is effectively a $2 \mathrm{D}$ honeycomb lattice with the two sublattices populated by metal atoms and the pairs of chalcogen atoms (Fig. 6a). In this structure, metal atoms are sixfold coordinated while chalcogen atoms are three-fold coordinated. Compared with the case of h-BN, however, these 2D materials are expected to display even greater structural variety of topological defects because of (i) the more easily changed coordination number of the constituent atoms, and (ii) the 3D character of their coordination spheres ${ }^{141,142}$. The binary chemical composition of h-BN and TMDCs also implies that topological defects in these materials realize different stoichiometries, making their formation energies dependent on the chemical potential. Potentially, the chemical degree of freedom presents another opportunity for engineering topological defects in 2D materials.

Several experimental studies of polycrystalline monolayer $\mathrm{MoS}_{2}$ have been published recently ${ }^{143-145}$. The samples have been produced by CVD growth starting from solid $\mathrm{MoO}_{3}$ and $\mathrm{S}$ precursors. This growth procedure results in triangular islands (single crystalline domains) of monolayer $\mathrm{MoS}_{2}$ with well-defined edges oriented either along Mo-rich or along S-rich zigzag directions (Fig. 6d). Growing 
a $\mathrm{h}-\mathrm{BN}$

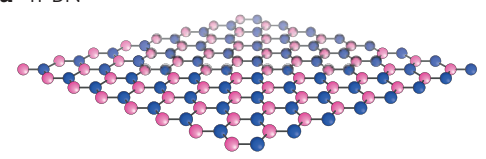

$\mathrm{MoS}_{2}$ and isostructural $\mathrm{MX}$, materials

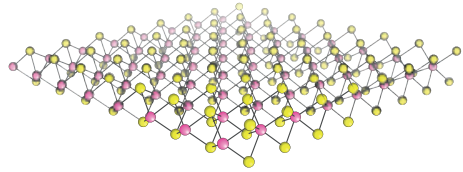

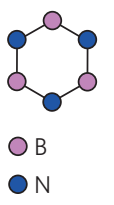

$\int_{0 \infty}^{\infty}$

Mo

OS b

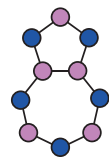

$\mathbf{b}=(1,0)$

B-rich

Neutral

$\mathrm{N}$-rich c

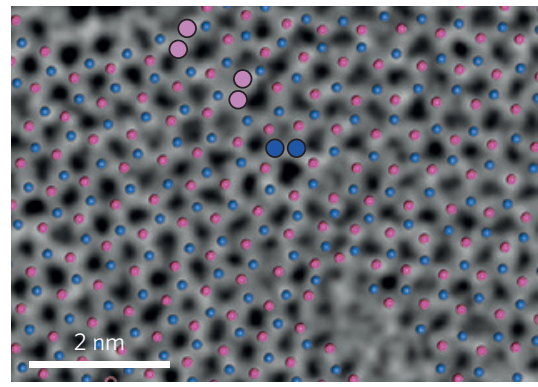

d
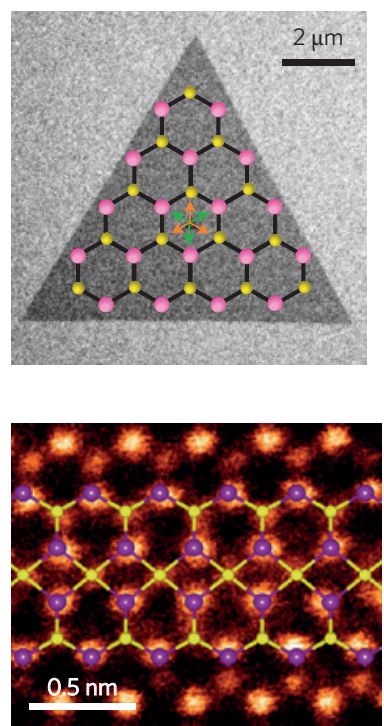

e

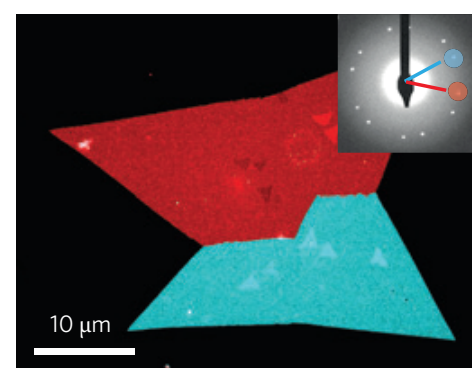

f

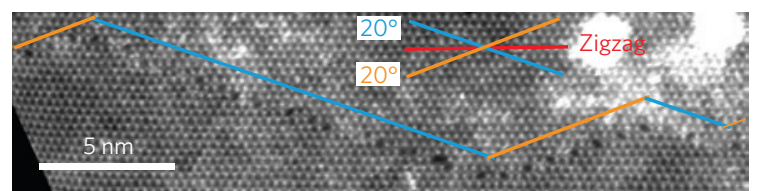

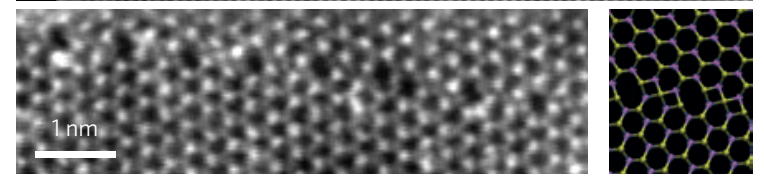
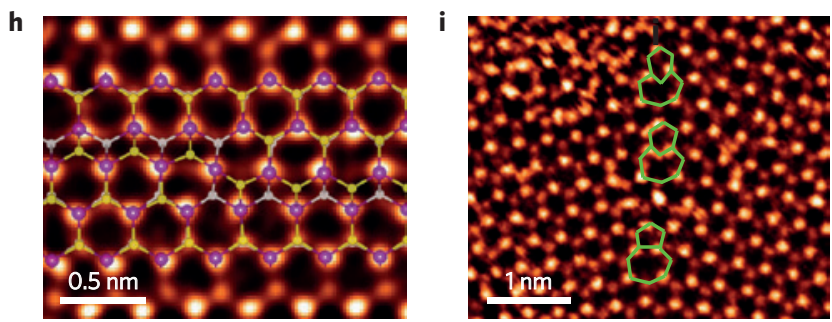

j

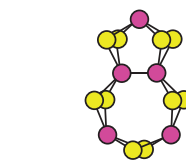

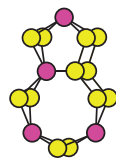
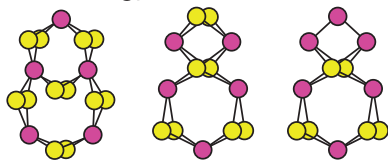

Figure 6 | Grain boundaries in binary two-dimensional materials. a, Structural models of binary two-dimensional materials: monolayer hexagonal boron nitride ( $h-B N$ ) and transition metal dichalcogenides ( $\mathrm{MoS}_{2}, \mathrm{WSe}_{2}$ and related materials). b, Proposed structures of dislocations in h-BN (ref. 139). The Burgers vector $\mathbf{b}=(1,0)$ dislocation cores composed of a pair of five- and seven-membered rings are charged and include a homoelemental bond. The neutral $\mathbf{b}=(1,1)$ dislocation core involves four- and eight-membered rings. $\mathbf{c}$, HR-TEM image of a grain boundary in h-BN showing the presence of both types of charged dislocation ${ }^{140}$. The atoms belonging to homoelemental bonds at dislocation cores are indicated by the larger coloured circles. $\mathbf{d}$, Single crystal of monolayer $\mathrm{MoS}_{2}$ with Mo-rich edges oriented along the zigzag direction. e, Dark-field TEM image of a polycrystalline aggregate of $\mathrm{MoS}_{2}$. The colour-coding corresponds to the diffraction spots indicated in the inset. f, Atomically resolved image (ADF-STEM) of a GB in MoS 2 reveals its highly faceted structure and preferential crystallographic orientation outlined by coloured lines ${ }^{143}$. The interface is composed of a regular arrangement of four- and eight-membered rings, as shown in the magnified image and structural model. $\mathbf{g}, \mathbf{h}$, Highly regular structures of $60^{\circ} \mathrm{GBs}$ in $\mathrm{MoS}_{2}$ observed in ref. 144. $\mathbf{i}$, High-resolution image (ADF-STEM) of an $18.5^{\circ} \mathrm{GB}$ consisting of dislocations with five- and seven-membered rings outlined by green lines. $\mathbf{j}$, Structural models of experimentally observed dislocation cores in monolayer $\mathrm{MoS}_{2}$ reported in refs 144 and 145. Figure reprinted with permission from: c, ref. 140, (c) American Chemical Society; d-f, ref. 143, Nature Publishing Group; g-i, ref. 144, (c) American Chemical Society.

islands inevitably form polycrystalline aggregates as exemplified in Fig. 6e. The images reported by van der Zande et al. reveal the highly faceted morphology of grain boundaries with their preferred crystallographic orientation inclined by around $20^{\circ}$ with respect to the zigzag direction ${ }^{143}$. At the atomic scale, the interface is composed of a regular arrangement of four- and eight-membered rings as shown in Fig. 6f. It was shown that such GBs have strong effects on optical properties and electrical conductivity consistent with the theoretical predictions of mid-gap states induced by such defects. Interestingly, somewhat different defect structures were reported by other authors in the samples of monolayer $\mathrm{MoS}_{2}$ produced by a very similar CVD process $^{144,145}$. The $60^{\circ}$ GBs were shown to be composed predominantly of four-membered rings arranged in two different patterns (Fig. 6g,h) ${ }^{144}$. Moreover, the observed small-angle GBs allowed individual $\mathbf{b}=(1,0)$ dislocations to be identified (Fig. 6i). Their great structural diversity, summarized in Fig. 6j, shows dislocation cores featuring four-membered rings, Mo-Mo and S-S bonds as well as undercoordinated S atoms.
Atomically thin silica constitutes another interesting example of hexagonal $2 \mathrm{D}$ lattice formed by corner-sharing $\mathrm{SiO}_{4}$ tetrahedra. TEM investigations of bilayer silica reveal the presence of topological and point defects similar to those observed in graphene ${ }^{146,147}$. Unlike graphene, however, 2D silica realizes an extra degree of structural freedom associated with the rotation of individual $\mathrm{SiO}_{4}$ units. This results in additional relaxation effects leading to very low formation energies of certain defects such as the large-angle GBs ${ }^{148}$. This peculiarity is ultimately reflected in the strong tendency of $2 \mathrm{D}$ silica to form a vitreous (amorphous) phase, which realizes the limit of high concentration of topological defects ${ }^{149,150}$.

\section{Perspectives}

This overview of recent progress highlights priority directions of research in the field of polycrystalline $2 \mathrm{D}$ materials. Although significant progress has been achieved in understanding the mechanism of CVD growth of graphene, much remains unclear about how graphene grains merge during the growth as their edges evolve and 
interact, and what defines the details of the atomic-scale structure of GBs formed. Much research effort will be devoted to developing methods of post-growth modification of polycrystalline graphene aimed at achieving desirable properties. Optimizing large-scale growth processes for increasing the size of single-crystalline graphene will remain one of the main vectors of research. However, tailoring graphene, its electrical, thermal, thermoelectric, mechanical and chemical properties, by means of purposefully introducing and manipulating topological disorder is expected to become another important objective. Atomic-precision engineering of individual topological defects to use them as components of new nanoscale devices sets the ultimate challenge for researchers. On the theory side, much remains to be done in terms of developing new tools for multiscale simulations of realistic models of polycrystalline $2 \mathrm{D}$ materials. Understanding the relations between the structural topological invariants of intrinsic defects in polycrystalline materials and their effects on various properties of these materials will continue to stimulate research. Finally, as the family of 2D materials continues expanding, the issues of their polycrystalline nature and related topological defects specific for each of these new materials will have to be addressed.

\section{Received 25 January 2014; accepted 15 July 2014;} published online 17 August 2014

\section{References}

1. Novoselov, K. S. et al. Electric field effect in atomically thin carbon films. Science 306, 666-669 (2004).

2. Novoselov, K. S. et al. Two-dimensional gas of massless Dirac fermions in graphene. Nature 438, 197-200 (2005).

3. Zhang, Y., Tan, Y-W., Stormer, H. L. \& Kim, P. Experimental observation of the quantum Hall effect and Berry's phase in graphene. Nature 438, 201-204 (2005).

4. Geim, A. K. \& Novoselov, K. S. The rise of graphene. Nature Mater. 6, 183-191 (2007).

5. Castro Neto, A. H., Guinea, F., Peres, N. M. R., Novoselov, K. S. \& Geim, A. K. The electronic properties of graphene. Rev. Mod. Phys. 81, 109-162 (2009).

6. Novoselov, K. S. Nobel Lecture. Graphene: Materials in the flatland. Rev. Mod. Phys. 83, 837-849 (2011).

7. Geim, A. K. Nobel Lecture. Random walk to graphene. Rev. Mod. Phys. 83, 851-862 (2011).

8. Schwierz, F. Graphene transistors. Nature Nanotech. 5, 487-496 (2010).

9. Bonaccorso, F., Sun, Z., Hasan, T. \& Ferrari, A. C. Graphene photonics and optoelectronics. Nature Photon. 4, 611-622 (2010).

10. Novoselov, K. S. et al. A roadmap for graphene. Nature 490, 192-200 (2012).

11. Golberg, D. et al. Boron nitride nanotubes and nanosheets. ACS Nano 4, 2979-2993 (2010).

12. Radisavljevic, B., Radenovic, A., Brivio, J., Giacometti, V. \& Kis, A. Single-layer $\mathrm{MoS}_{2}$ transistors. Nature Nanotech. 6, 147-150 (2011).

13. Wang, Q. H., Kalantar-Zadeh, K., Kis, A., Coleman, J. N. \& Strano, M. S. Electronics and optoelectronics of two-dimensional transition metal dichalcogenides. Nature Nanotech. 7, 699-712 (2012).

14. Mayorov, A. S. et al. Micrometer-scale ballistic transport in encapsulated graphene at room temperature. Nano Lett. 11, 2396-2399 (2011).

15. Britnell, L. et al. Field-effect tunneling transistor based on vertical graphene heterostructures. Science 335, 947-950 (2012).

16. Georgiou, T. et al. Vertical field-effect transistor based on graphene-WS heterostructures for flexible and transparent electronics. Nature Nanotech. 8, 100-103 (2013).

17. Mermin, N. D. Crystalline order in two dimensions. Phys. Rev. 176, 250-254 (1968).

18. Huang, P. Y. et al. Grains and grain boundaries in single-layer graphene atomic patchwork quilts. Nature 469, 389-392 (2011).

This is the first paper reporting systematic experimental investigation of polycrystalline graphene including atomic structure of grain boundaries.

19. Kim, K. et al. Grain boundary mapping in polycrystalline graphene. ACS Nano 5, 2142-2146 (2011).

20. An, J. et al. Domain (grain) boundaries and evidence of 'twinlike' structures in chemically vapor deposited grown graphene. ACS Nano 5, 2433-2439 (2011).

21. Hirth, J. P. \& Lothe, J. Theory of Dislocations (McGraw-Hill, 1968).

22. Sutton, A. P. \& Balluff, R. W. Interfaces in Crystalline Materials (Clarendon, 1995).
23. Nelson, D. R. Defects and Geometry in Condensed Matter Physics (Cambridge Univ. Press, 2002).

24. Amelinckx, S. \& Delavignette, P. Dislocation loops due to quenched-in point defects in graphite. Phys. Rev. Lett. 5, 50-51 (1960).

25. Delavignette, P. \& Amelinckx, S. Dislocation patterns in graphite. J. Nucl. Mater. 5, 17-66 (1962).

26. Roscoe, C. \& Thomas, J. M. The revelation of small-angle boundaries and forest dislocations in graphite monocrystals. Carbon 4, 383-390 (1966).

27. Albrecht, T. R., Mizes, H. A., Nogami, J., Park, S-i. \& Quate, C. F. Observation of tilt boundaries in graphite by scanning tunneling microscopy and associated multiple tip effects. Appl. Phys. Lett. 52, 362-364 (1988).

28. Clemmer, C. \& Beebe, T. Jr Graphite: a mimic for DNA and other biomolecules in scanning tunneling microscope studies. Science 251, 640-642 (1991).

29. Heckl, W. M. \& Binnig, G. Domain walls on graphite mimic DNA. Ultramicroscopy 42, 1073-1078 (1992).

30. Simonis, P. et al. STM study of a grain boundary in graphite. Surf. Sci. 511, 319-322 (2002)

31. Červenka, J. \& Flipse, C. F. J. Structural and electronic properties of grain boundaries in graphite: Planes of periodically distributed point defects. Phys. Rev. B 79, 195429 (2009).

32. Červenka, J., Katsnelson, M. I. \& Flipse, C. F. J. Room-temperature ferromagnetism in graphite driven by two-dimensional networks of point defects. Nature Phys. 5, 840-844 (2009).

33. Robertson, A. W. \& Warner, J. H. Atomic resolution imaging of graphene by transmission electron microscopy. Nanoscale 5, 4079 (2013).

34. Hashimoto, A., Suenaga, K., Gloter, A., Urita, K. \& Iijima, S. Direct evidence for atomic defects in graphene layers. Nature 430, 870-873 (2004).

35. Li, X. et al. Large-area synthesis of high-quality and uniform graphene films on copper foils. Science 324, 1312-1314 (2009).

This paper reports the chemical vapour deposition synthesis of large-area monolayer graphene on $\mathrm{Cu}$ foil.

36. Yazyev, O. V. \& Louie, S. G. Topological defects in graphene: Dislocations and grain boundaries. Phys. Rev. B 81, 195420 (2010).

This theoretical paper reports a model of the structure of topological defects in polycrystalline graphene.

37. Liu, Y. \& Yakobson, B. I. Cones, pringles, and grain boundary landscapes in graphene topology. Nano Lett. 10, 2178-2183 (2010).

38. Read, W. T. \& Shockley, W. Dislocation models of crystal grain boundaries. Phys. Rev. 78, 275-289 (1950).

39. Seung, H. S. \& Nelson, D. R. Defects in flexible membranes with crystalline order. Phys. Rev. A 38, 1005-1018 (1988).

40. Carraro, C. \& Nelson, D. R. Grain-boundary buckling and spin-glass models of disorder in membranes. Phys. Rev. E 48, 3082-3090 (1993).

41. Malola, S., Häkkinen, H. \& Koskinen, P. Structural, chemical, and dynamical trends in graphene grain boundaries. Phys. Rev. B 81, 165447 (2010).

42. Akhukov, M. A., Fasolino, A., Gornostyrev, Y. N. \& Katsnelson, M. I. Dangling bonds and magnetism of grain boundaries in graphene. Phys. Rev. B 85, 115407 (2012).

43. Robertson, A. W. et al. Atomic structure of interconnected few-layer graphene domains. ACS Nano 5, 6610-6618 (2011).

44. Tsen, A. W. et al. Tailoring electrical transport across grain boundaries in polycrystalline graphene. Science 336, 1143-1146 (2012).

45. Lahiri, J., Lin, Y., Bozkurt, P., Oleynik, I. I. \& Batzill, M. An extended defect in graphene as a metallic wire. Nature Nanotech. 5, 326-329 (2010).

46. Chen, J. H. et al. Controlled growth of a line defect in graphene and implications for gate-tunable valley filtering. Phys. Rev. B 89, 121407 (2014).

47. Warner, J. H. et al. Bond length and charge density variations within extended arm chair defects in graphene. ACS Nano 7, 9860-9866 (2013).

48. Cockayne, E. et al. Grain boundary loops in graphene. Phys. Rev. B 83, 195425 (2011).

49. Meyer, J. C. et al. Experimental analysis of charge redistribution due to chemical bonding by high-resolution transmission electron microscopy. Nature Mater. 10, 209-215 (2011).

50. Kotakoski, J., Krasheninnikov, A. V., Kaiser, U. \& Meyer, J. C. From point defects in graphene to two-dimensional amorphous carbon. Phys. Rev. Lett. 106, 105505 (2011).

51. Kotakoski, J. et al. Stone-Wales-type transformations in carbon nanostructures driven by electron irradiation. Phys. Rev. B 83, 245420 (2011).

52. Kurasch, S. et al. Atom-by-atom observation of grain boundary migration in graphene. Nano Lett. 12, 3168-3173 (2012)

53. Brown, L., Hovden, R., Huang, P., Wojcik, M., Muller, D. A. \& Park, J. Twinning and twisting of tri- and bilayer graphene. Nano Lett. 12, 1609-1615 (2012)

54. Alden, J. S. et al. Strain solitons and topological defects in bilayer graphene. Proc. Natl Acad. Sci. USA 110, 11256-11260 (2013). 
55. Lin, J. H. et al. AC/AB stacking boundaries in bilayer graphene. Nano Lett. 13, 3262-3268 (2013)

56. Gong, L. et al. Reversible loss of Bernal stacking during the deformation of few-layer graphene in nanocomposites. ACS Nano 7, 7287-7294 (2013).

57. Butz, B. et al. Dislocations in bilayer graphene. Nature 505, 533-537 (2014).

58. Carlsson, J. M., Ghiringhelli, L. M. \& Fasolino, A. Theory and hierarchical calculations of the structure and energetics of [0001] tilt grain boundaries in graphene. Phys. Rev. B 84, 165423 (2011).

59. Liu, T-H., Gajewski, G., Pao, C-W. \& Chang, C-C. Structure, energy, and structural transformations of graphene grain boundaries from atomistic simulations. Carbon 49, 2306-2317 (2011).

60. Chen, S. \& Chrzan, D. C. Continuum theory of dislocations and buckling in graphene. Phys. Rev. B 84, 214103 (2011).

61. Li, L., Reich, S. \& Robertson, J. Defect energies of graphite: Density-functional calculations. Phys. Rev. B 72, 184109 (2005).

62. Coraux, J., N’Diaye, A. T., Busse, C. \& Michely, T. Structural coherency of graphene on $\operatorname{Ir}(111)$. Nano Lett. 8, 565-570 (2008).

63. Lehtinen, O., Kurasch, S., Krasheninnikov, A. V. \& Kaiser, U. Atomic scale study of the life cycle of a dislocation in graphene from birth to annihilation. Nature Commun. 4, 2098 (2013).

64. Warner, J. H. et al. Rippling graphene at the nanoscale through dislocation addition. Nano Lett. 13, 4937-4944 (2013).

65. Yazyev, O. V. \& Louie, S. G. Electronic transport in polycrystalline graphene. Nature Mater. 9, 806-809 (2010).

This is the first theoretical paper describing transport properties of grain boundaries in graphene.

66. Capasso, A. et al. Graphene ripples generated by grain boundaries in highly ordered pyrolytic graphite. Carbon 68, 330-336 (2014)

67. Warner, J. H. et al. Dislocation-driven deformations in graphene. Science 337, 209-212 (2012).

68. Yakobson, B. I. Mechanical relaxation and 'intramolecular plasticity' in carbon nanotubes. Appl. Phys. Lett. 72, 918-920 (1998).

69. Ding, F., Jiao, K., Wu, M. \& Yakobson, B. I. Pseudoclimb and dislocation dynamics in superplastic nanotubes. Phys. Rev. Lett. 98, 075503 (2007).

70. Ding, F., Jiao, K., Lin, Y. \& Yakobson, B. I. How evaporating carbon nanotubes retain their perfection? Nano Lett. 7, 681-684 (2007).

71. Banhart, F. Irradiation effects in carbon nanostructures. Rep. Prog. Phys. 62, 1181-1221 (1999)

72. Meyer, J. C. et al. Accurate measurement of electron beam induced displacement cross sections for single-layer graphene. Phys. Rev. Lett. 108, 196102 (2012).

73. Bae, S. et al. Roll-to-roll production of 30-inch graphene films for transparent electrodes. Nature Nanotech. 5, 574-578 (2010).

74. Zhang, W., Wu, P., Li, Z. \& Yang, J. First-principles thermodynamics of graphene growth on Cu surfaces. J. Phys. Chem. C 115, 17782-17787 (2011).

75. Kim, H. et al. Activation energy paths for graphene nucleation and growth on Cu. ACS Nano 6, 3614-3623 (2012).

76. Yu, Q. et al. Control and characterization of individual grains and grain boundaries in graphene grown by chemical vapour deposition. Nature Mater. 10, 443-449 (2011)

This paper reports the first experimental measurements of electronic transport across isolated grain boundaries in graphene.

77. Hao, Y. et al. The role of surface oxygen in the growth of large single-crystal graphene on copper. Science 342, 720-723 (2013).

This work reveals the role of surface oxygen in chemical vapour deposition growth of graphene.

78. Gao, L., Guest, J. R. \& Guisinger, N. P. Epitaxial graphene on $\mathrm{Cu}(111)$. Nano Lett. 10, 3512-3516 (2010).

79. Nie, S., Wofford, J. M., Bartelt, N. C., Dubon, O. D. \& McCarty, K. F. Origin of the mosaicity in graphene grown on $\mathrm{Cu}(111)$. Phys. Rev. B 84, 155425 (2011)

80. Wood, J. D., Schmucker, S. W., Lyons, A. S., Pop, E. \& Lyding, J. W. Effects of polycrystalline cu substrate on graphene growth by chemical vapor deposition. Nano Lett. 11, 4547-4554 (2011).

81. Ogawa, Y. et al. Domain structure and boundary in single-layer graphene grown on $\mathrm{Cu}(111)$ and $\mathrm{Cu}(100)$ films. J. Phys. Chem. Lett. 3, 219-226 (2012).

82. Murdock, A. T. et al. Controlling the orientation, edge geometry, and thickness of chemical vapor deposition graphene. ACS Nano 7, 1351-1359 (2013).

83. Meca, E., Lowengrub, J., Kim, H., Mattevi, C. \& Shenoy, V. B. Epitaxial graphene growth and shape dynamics on copper: Phase-field modeling and experiments. Nano Lett. 13, 5692-5697 (2013)

84. Han, G. H. et al. Influence of copper morphology in forming nucleation seeds for graphene growth. Nano Lett. 11, 4144-4148 (2011)

85. Yan, Z. et al. Toward the synthesis of wafer-scale single-crystal graphene on copper foils. ACS Nano 6, 9110-9117 (2012).

86. Zhou, H. L. et al. Chemical vapour deposition growth of large single crystals of monolayer and bilayer graphene. Nature Commun. 4, 2096 (2013).
87. Mohsin, A. et al. Synthesis of millimeter-size hexagon-shaped graphene single crystals on resolidified copper. ACS Nano 7, 8924 (2013).

88. Wu, W. et al. Growth of single crystal graphene arrays by locally controlling nucleation on polycrystalline $\mathrm{Cu}$ using chemical vapor deposition. Adv. Mater. 23, 4898-4903 (2011)

89. Massicotte, M., Yu, V., Whiteway, E., Vatnik, D. \& Hilke, M. Quantum Hall effect in fractal graphene: growth and properties of graphlocons. Nanotechnology 24, 325601 (2013).

90. Wu, B. et al. Self-organized graphene crystal patterns. NPG Asia Mater. 5, e36 (2013).

91. Tian, J., Cao, H., Wu, W., Yu, Q. \& Chen, Y. P. Direct imaging of graphene edges: Atomic structure and electronic scattering. Nano Lett. 11, 3663-3668 (2011)

92. Vlassiouk, I. et al. Role of hydrogen in chemical vapor deposition growth of large single-crystal graphene. ACS Nano 5, 6069-6076 (2011).

93. Vlassiouk, I. et al. Graphene nucleation density on copper: Fundamental role of background pressure. J. Phys. Chem. C 117, 18919-18926 (2013).

94. Artyukhov, V. I., Liu, Y. \& Yakobson, B. I. Equilibrium at the edge and atomistic mechanisms of graphene growth. Proc. Natl Acad. Sci. USA 109, 15136-15140 (2012).

95. Ma, T. et al. Edge-controlled growth and kinetics of single-crystal graphene domains by chemical vapor deposition. Proc. Natl. Acad. Sci. USA 110, 20386-20391 (2013).

96. Kim, D. W., Kim, Y. H., Jeong, H. S. \& Jung, H-T. Direct visualization of large-area graphene domains and boundaries by optical birefringency. Nature Nanotech. 7, 29-34 (2012).

97. Duong, D. L. et al. Probing graphene grain boundaries with optical microscopy. Nature 490, 235-239 (2012).

98. Jia, C. et al. Direct optical characterization of graphene growth and domains on growth substrates. Sci. Rep. 2, 707 (2012).

99. Nemes-Incze, P. et al. Revealing the grain structure of graphene grown by chemical vapor deposition. Appl. Phys. Lett. 99, 023104 (2011).

100. Fei, Z. et al. Electronic and plasmonic phenomena at graphene grain boundaries. Nature Nanotech. 8, 821-825 (2013).

101. Li, X. et al. Graphene films with large domain size by a two-step chemical vapor deposition process. Nano Lett. 10, 4328-4334 (2010).

102. Song, H. S. et al. Origin of the relatively low transport mobility of graphene grown through chemical vapor deposition. Sci. Rep. 2, 337 (2012).

103. Tapaszto, L. et al. Mapping the electronic properties of individual graphene grain boundaries. Appl. Phys. Lett. 100, 053114 (2012).

104. Ahmad, M. et al. Nanoscale investigation of charge transport at the grain boundaries ad wrinkles in graphene film. Nanotechnology 23, 285705 (2012)

105. Jauregui, L. A., Cao, H., Wu, W., Yu, Q. \& Chen, Y. P. Electronic properties of grains and grain boundaries in graphene grown by chemical vapor deposition. Solid State Commun. 151, 1100-1104 (2011)

106. Petrone, N. et al. Chemical vapor deposition-derived graphene with electrical performance of exfoliated graphene. Nano Lett. 12, 2751-2756 (2012).

107. Ryu, J. et al. Fast synthesis of high-performance graphene films by hydrogen free rapid thermal chemical vapor deposition. ACS Nano 8, 950-956 (2013).

108. Cançado, L. G., Pimenta, M. A., Neves, B. R. A., Dantas, M. S. S. \& Jorio, A. Influence of the atomic structure on the Raman spectra of graphite edges. Phys. Rev. Lett. 93, 247401 (2004).

109. Ferrari, A. C. Raman spectroscopy of graphene and graphite: Disorder, electron-phonon coupling, doping and nonadiabatic effects. Solid State Commun. 143, 47-57 (2007).

110. Shen, T. et al. Quantum Hall effect on centimeter scale chemical vapor deposited graphene films. Appl. Phys. Lett. 99, 232110 (2011).

111. Clark, K. W. et al. Spatially resolved mapping of electrical conductivity across individual domain (grain) boundaries in graphene. ACS Nano 7, 7956-7966 (2013)

112. Koepke, J. C. et al. Atomic-scale evidence for potential barriers and strong carrier scattering at graphene grain boundaries: A scanning tunneling microscopy study. ACS Nano 7, 75-86 (2012)

113. Büttiker, M., Imry, Y., Landauer, R. \& Pinhas, S. Generalized manychannel conductance formula with application to small rings. Phys. Rev. B 31, 6207-6215 (1985).

114. Gunlycke, D. \& White, C. T. Graphene valley filter using a line defect. Phys. Rev. Lett. 106, 136806 (2011).

115. Rycerz, A., Tworzydlo, J. \& Beenakker, C. W. J. Valley filter and valley valve in graphene. Nature Phys. 3, 172-175 (2007).

116. Akhmerov, A. R. \& Beenakker, C. W. J. Detection of valley polarization in graphene by a superconducting contact. Phys. Rev. Lett. 98, 157003 (2007)

117. Xiao, D., Yao, W. \& Niu, Q. Valley-contrasting physics in graphene: Magnetic moment and topological transport. Phys. Rev. Lett. 99, 236809 (2007).

118. Kumar, S. B. \& Guo, J. Strain-induced conductance modulation in graphene grain boundary. Nano Lett. 12, 1362-1366 (2012) 
119. Gunlycke, D., Vasudevan, S. \& White, C. T. Confinement, transport gap, and valley polarization in graphene from two parallel decorated line defects. Nano Lett. 13, 259-263 (2012).

120. Zhang, H., Lee, G., Gong, C., Colombo, L. \& Cho, K. Grain boundary effect on electrical transport properties of graphene. J. Phys. Chem. C 118, 2338-2343 (2014).

121. Vancsó, P. et al. Electronic transport through ordered and disordered graphene grain boundaries. Carbon 64, 101-110 (2013).

122. Van Tuan, D. et al. Scaling properties of charge transport in polycrystalline graphene. Nano Lett. 13, 1730-1735 (2013).

123. Gargiulo, F. \& Yazyev, O. V. Topological aspects of charge-carrier transmission across grain boundaries in graphene. Nano Lett. 14, 250-254 (2014).

124. Cortijo, A. \& Vozmediano, M. A. H. Effects of topological defects and local curvature on the electronic properties of planar graphene. Nucl. Phys. B 763, 293-308 (2007).

125. Mesaros, A., Sadri, D. \& Zaanen, J. Berry phase of dislocations in graphene and valley conserving decoherence. Phys. Rev. B 79, 155111 (2009).

126. Mesaros, A., Papanikolaou, S., Flipse, C. F. J., Sadri, D. \& Zaanen, J. Electronic states of graphene grain boundaries. Phys. Rev. B 82, 205119 (2010).

127. Meyers, M. A. \& Chawla, K. K. Mechanical Behavior of Materials (Cambridge Univ. Press, 2009).

128. Lee, C., Wei, X., Kysar, J. W. \& Hone, J. Measurement of the elastic properties and intrinsic strength of monolayer graphene. Science 321, 385-388 (2008).

129. Lee, G-H. et al. High-strength chemical-vapor-deposited graphene and grain boundaries. Science 340, 1073-1076 (2013).

This experimental work shows that mechanical properties of polycrystalline graphene compare to those of single crystalline samples.

130. Ruiz-Vargas, C. S. et al. Softened elastic response and unzipping in chemical vapor deposition graphene membranes. Nano Lett. 11, 2259-2263 (2011)

131. Grantab, R., Shenoy, V. B. \& Ruoff, R. S. Anomalous strength characteristics of tilt grain boundaries in graphene. Science 330, 946-948 (2010).

132. Wei, Y. et al. The nature of strength enhancement and weakening by pentagon-heptagon defects in graphene. Nature Mater. 11, 759-763 (2012).

133. Rasool, H. I., Ophus, C., Klug, W. S., Zettl, A. \& Gimzewski, J. K. Measurement of the intrinsic strength of crystalline and polycrystalline graphene. Nature Commun. 4, 2811 (2013).

134. Kotakoski, J. \& Meyer, J. C. Mechanical properties of polycrystalline graphene based on a realistic atomistic model. Phys. Rev. B 85, 195447 (2012).

135. Song, Z., Artyukhov, V. I., Yakobson, B. I. \& Xu, Z. Pseudo Hall-Petch strength reduction in polycrystalline graphene. Nano Lett. 13, 1829-1833 (2013).

136. Cao, A. \& Qu, J. Atomistic simulation study of brittle failure in nanocrystalline graphene under uniaxial tension. Appl. Phys. Lett. 102, 071902 (2013).

137. Bagri, A., Kim, S-P., Ruoff, R. S. \& Shenoy, V. B. Thermal transport across twin grain boundaries in polycrystalline graphene from nonequilibrium molecular dynamics simulations. Nano Lett. 11, 3917-3921 (2011).

138. Wang, Y., Vallabhaneni, A. K., Qiu, B. \& Ruan, X. Two-dimensional thermal transport in graphene: a review of numerical modeling studies. Nanoscale Microscale Thermophys. Eng. 18, 155-182 (2014).

139. Liu, Y., Zou, X. \& Yakobson, B. I. Dislocations and grain boundaries in twodimensional boron nitride. ACS Nano 6, 7053-7058 (2012).
140. Gibb, A. L. et al. Atomic resolution imaging of grain boundary defects in monolayer chemical vapor deposition-grown hexagonal boron nitride. J. Am. Chem. Soc. 135, 6758-6761 (2013)

141. Zou, X., Liu, Y. \& Yakobson, B. I. Predicting dislocations and grain boundaries in two-dimensional metal-disulfides from the first principles. Nano Lett. 13, 253-258 (2012).

This theoretical work predicts topological defects in monolayer transition metal dichalcogenides and shows their structural and stoichiometric diversity.

142. Zhang, Z., Zou, X., Crespi, V. H. \& Yakobson, B. I. Intrinsic magnetism of grain boundaries in two-dimensional metal dichalcogenides. ACS Nano 7, 10475-10481 (2013).

143. van der Zande, A. M. et al. Grains and grain boundaries in highly crystalline monolayer molybdenum disulphide. Nature Mater. 12, 554-561 (2013).

This is the first (shortly followed by a few other) experimental study of polycrystalline monolayer $\mathrm{MoS}_{2}$.

144. Zhou, W. et al. Intrinsic structural defects in monolayer molybdenum disulfide. Nano Lett. 13, 2615-2622 (2013).

145. Najmaei, S. et al. Vapour phase growth and grain boundary structure of molybdenum disulphide atomic layers. Nature Mater. 12, 754-759 (2013).

146. Huang, P. Y. et al. Imaging atomic rearrangements in two-dimensional silica glass: watching silica's dance. Science 342, 224-227 (2013).

147. Yang, B., Boscoboinik, J. A., Yu, X., Shaikhutdinov, S. \& Freund, H-J. Patterned defect structures predicted for graphene are observed on single-layer silica films. Nano Lett. 13, 4422-4427 (2013).

148. Björkman, T. et al. Defects in bilayer silica and graphene: common trends in diverse hexagonal two-dimensional systems. Sci. Rep. 3, 3482 (2013).

149. Lichtenstein, L., Heyde, M. \& Freund, H-J. Crystalline-vitreous interface in two dimensional silica. Phys. Rev. Lett. 109, 106101 (2012).

150. Lichtenstein, L. et al. The atomic structure of a metal-supported vitreous thin silica film. Angew. Chem. Int. Ed. 51, 404-407 (2012).

\section{Acknowledgements}

O.V.Y. acknowledges funding from the Swiss NSF (grant no. PP00P2_133552) and the ERC for Grant “TopoMat” (no. 306504), as well as technical assistance from G. Autès and F. Gargiulo while preparing the manuscript. Y.P.C. acknowledges research support from NSF, NIST, DTRA, DHS and Purdue University, and collaborations and helpful discussions with many group members and colleagues on topics related to this Review, in particular H. Cao, T.-F. Chung, R. Colby, L. A. Jauregui, E. A. Stach, J. Tian and Q. Yu.

\section{Additional information}

Reprints and permissions information is available online at www.nature.com/reprints. Correspondence and requests for materials should be addressed to O.V.Y. and Y.P.C.

\section{Competing financial interests}

The authors declare no competing financial interests. 